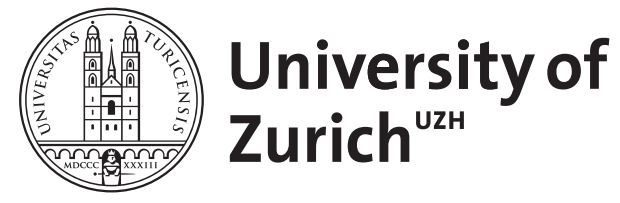

Zurich Open Repository and Archive

University of Zurich

University Library

Strickhofstrasse 39

CH-8057 Zurich

www.zora.uzh.ch

Year: 2007

\title{
Reform des internationalen Versicherungsvertragsrechts
}

Heiss, Helmut

DOI: https://doi.org/10.1007/bf03353548

Posted at the Zurich Open Repository and Archive, University of Zurich

ZORA URL: https://doi.org/10.5167/uzh-155735

Journal Article

Published Version

Originally published at:

Heiss, Helmut (2007). Reform des internationalen Versicherungsvertragsrechts. Zeitschrift für die gesamte Versicherungswissenschaft (ZVersWiss), 96(4):503-534.

DOI: https://doi.org/10.1007/bf03353548 


\title{
Reform des internationalen Versicherungsvertragsrechts
}

\author{
Von Helmut Heis s, Zürich ${ }^{1}$
}

\section{Inhaltsübersicht}

1. Sonderstellung und Defizite des geltenden internationalen Versicherungsvertragsrechts

2. Reformvorschläge von Kommission, Parlament und Rat

3. Reformvorschlag des Max-Planck-Instituts für ausländisches und internationales Privatrecht vom 16. Juni $2006^{2}$, Hamburg

4. Eigener Vorschlag

\section{Sonderstellung und Defizite des geltenden internationalen Versicherungsvertragsrechts}

\subsection{Zur Sonderstellung des internationalen Versicherungsvertragsrechts im europäischen Kollisionsrecht}

Das internationale Versicherungsvertragsrecht nimmt im europäischen Kollisionsrecht seit jeher eine markante Sonderstellung ein. Im Brüsseler Übereinkommen über die gerichtliche Zuständigkeit und die Anerkennung und Vollstreckung von Entscheidungen in Zivil- und Handelssachen von $1968^{3}$ bildet das Zuständigkeitsrecht für Versicherungssachen zwar einen integralen Bestandteil des Rechtsakts, doch wird es in einem eigenen Abschnitt 3 (Art. 7-12a EuGVÜ) geregelt, der sich als eine abschließende Regelung begreift und daher einen Rückgriff auf die allgemeinen Zuständigkeitsvorschriften nur dort erlaubt, wo auf diese besonders Bezug genommen wird. Diese Sonderstellung haben sich die Zuständigkeitsregeln für Versicherungssachen auch im späteren Luganer Übereinkommen ${ }^{4}$ und

1 Prof. Dr. iur. Helmut Heiss, Lehrstuhl für Privatrecht, Rechtsvergleichung und IPR, Universität Zürich.

2 Max Planck Institute for Comparative and International Private Law, Comments on the European Commission's Proposal for a Regulation of the European Parliament and the Council on the law applicable to contractual obligations (Rome I), RabelsZ 71 (2007) 225; einsehbar auch unter http://www.mpipriv-hh.mpg.de; im Folgenden zitiert als: MPI-Vorschlag, RabelsZ 71 (2007) 225.

3 Brüsseler Übereinkommen vom 27. 9. 1968, ABl. 1972 Nr. L 299 / 32 i. d. g. F.

4 Luganer Übereinkommen vom 16. 9. 1988, ABl. 1988 Nr. L 319 / 9 i. d. g. F. 
zuletzt in der Verordnung (EG) Nr. $44 / 2001^{5}$ des Rates über die gerichtliche Zuständigkeit und die Anerkennung und Vollstreckung von Entscheidungen in Zivil- und Handelssachen (Art. 8-14) voll umfänglich bewahrt.

Wesentlich drastischer noch als im Recht der internationalen Zuständigkeit zeigt sich die Sonderstellung des internationalen Versicherungsvertragsrechts im europäischen internationalen Privatrecht. Das Römische EWG-Übereinkommen über das auf vertragliche Schuldverhältnisse anzuwendende Recht von $1980^{6}$ regelt lediglich Rückversicherungsverträge sowie Erstversicherungsverträge über Risiken, die nicht im Hoheitsgebiet eines Mitgliedstaats der Europäischen Gemeinschaft belegen sind. ${ }^{7}$ Versicherungsverträge mit Binnenmarktbezug, also Verträge über Risiken, die im Hoheitsgebiet eines Mitgliedstaats der EG belegen sind, bleiben dagegen ausgenommen. Die Wissenschaft hat diese Ausklammerung seit jeher bedauert. ${ }^{8}$ Offenbar aber konnten sich die Vertragsstaaten des EVÜ nicht auf eine einheitliche Regelung für Versicherungsverträge einigen. Mit Blick auf die damals ohnehin laufenden Arbeiten des EG-Gesetzgebers an einer Harmonisierung des Versicherungsrechts ließen die Vertragsstaaten des EVÜ die binnenmarktbezogenen Versicherungsverträge schließlich ungeregelt. ${ }^{9}$ Der Verweis auf die geplanten Rechtssetzungsschritte des europäischen Gesetzgebers dürfte sich dabei vor allem auf jene Vorarbeiten bezogen haben, die in den Kommissionsvorschlag einer Richtlinie zur Koordinierung des Versicherungsvertragsrechts aus 1979/1980 mündeten. ${ }^{10}$ Dieser Vor-

5 Verordnung (EG) Nr. 44 / 2001 vom 22. 12.2000, ABl. 2001 Nr. L 12 / 1.

6 Römisches Übereinkommen vom 19. 6. 1980 ABl. 1980 Nr. L 266 / 1; konsolidierte Fassung nach dem 4. Beitrittsübereinkommen in ABl. 2005 Nr. C $334 / 3$.

7 Hierzu aus der Sicht deutschen Rechts E. Lorenz, Zum neuen internationalen Vertragsrecht aus versicherungsvertraglicher Sicht, in: FS Kegel (1987) 303.

8 Lando, The EC Draft Convention on the Law Applicable to Contractual and NonContractual Obligations, RabelsZ 38 (1974) 6 (10); die Schaffung des Kollisionsrechts in den Richtlinien hat diese Kritik nicht beseitigt; vgl. Schnyder, Parteiautonomie im europäischen Versicherungskollisionsrecht, in: Reichert-Facilides (Hrsg.), Aspekte des internationalen Versicherungsvertragsrechts im Europäischen Wirtschaftsraum (1994) 49 (52 f.); vgl. auch - aus der Sicht deutschen Rechts - Fricke, Die Neuregelung des IPR der Versicherungsverträge im EGVVG durch das Gesetz zur Durchführung versicherungsrechtlicher Richtlinien in der EG, IPRax 1990, 361 (364).

9 Siehe den Bericht über das Übereinkommen über das auf vertragliche Schuldverhältnisse anzuwendende Recht von Giuliano/Lagarde, ABl 1980 Nr. C 282/1 zu Art. 1 Abs. 3 EVÜ; Lando, The EC Draft Convention on the Law Applicable to Contractual and Non-Contractual Obligations, RabelsZ 38 (1974) 6 (10).

10 Vorschlag für eine Richtlinie des Rates zur Koordinierung der Rechts- und Verwaltungsvorschriften betreffend die Aufnahme und Ausübung der Tätigkeit der Direktversicherung, ABl. 1979 Nr. C 190/2; zum Verhältnis dieses Richtlinien-Vorschlags zum EVÜ z. B. Pearson, Opening Address, in: Reichert-Facilides / d'Oliveira (eds.), International Insurance Contract Law in the EC (1993)1 (3); zum Richtlinienvorschlag aus heutiger Sicht Basedow, Die Gesetzgebung zum Versicherungsvertrag zwischen europäischer Integration und Verbraucherpolitik, in: Reichert-Facilides / Schnyder (Hrsg.), Versicherungsrecht in Europa - Kernperspektiven am Ende des 20. Jahrhunderts, ZSR 2000, Beiheft 34, 13 (15 ff.). 
schlag zur Angleichung des materiellen Versicherungsvertragsrechts ist indessen gescheitert. Der Handlungsdruck aber blieb auf dem europäischen Gesetzgeber lasten und verstärkte sich, als der EuGH im Dezember 1986 seine Urteile ${ }^{11}$ zur Dienstleistungsfreiheit im Versicherungssektor sprach. ${ }^{12}$ Der EG-Gesetzgeber beschloss, neben der Verwirklichung eines Herkunftslandprinzips im Aufsichtsrecht auch das internationale Versicherungsvertragsrecht $\mathrm{zu}$ harmonisieren. ${ }^{13}$ Offenbar stand hinter diesem Vorhaben die Idee, ein angeglichenes Kollisionsrecht könne den Versicherungsbinnenmarkt auch ohne Harmonisierung des materiellen Versicherungsvertragsrechts verwirklichen. ${ }^{14}$ So enthalten die zweite und die dritte Generation der Richtlinien zum Versicherungsrecht nicht zuletzt auch das Binnenmarktkollisionsrecht für Versicherungen. ${ }^{15}$ Aufgrund des mittlerweile geschlossenen EWR-Abkommens reicht der geographische Geltungsbereich dieser Richtlinien über die EG-Mitgliedstaaten hinaus und umfasst alle EWR-Mitgliedstaaten. ${ }^{16}$ Die Richtlinien entziehen damit dem EVÜ einen Teil seines Anwendungsbereichs, weil das Richtlinienrecht und seine nationalen Umsetzungen gemäß Art. 20 EVÜ Vorrang genießen. ${ }^{17}$

Nachfolgende Rechtsakte haben das internationale Versicherungsvertragsrecht zwar beeinflusst, nicht aber grundlegend verändert, insbesondere nicht seine Sonderstellung beseitigt. So haben die Richtlinien zum Verbrauchervertragsrecht, die zum Teil auch auf Versicherungsverträge zur Anwendung kommen, das internationale Verbrauchervertragsrecht des Art. 5

11 EuGH 4. 12.1986 (RS C-205 / 84) Slg. 1986, 3755 (Kommission ./ . BRD); EuGH 4. 12.1986 (RS C-252 / 83) Slg. 1986, 3713 (Kommission ./. Dänemark); EuGH 4. 12. 1986 (C-220/83) Slg. 1986, 3663 (Kommission ./. Frankreich); EuGH 4. 12. 1986 (C-206 / 84) Slg. 1986, 3817 (Kommission ./. Irland).

12 Zum Problem des Zeitdrucks bei der Verwirklichung des Versicherungsbinnenmarktes Basedow, Die Gesetzgebung zum Versicherungsvertrag zwischen europäischer Integration und Verbraucherpolitik, in: Reichert-Facilides / Schnyder (Hrsg.), Versicherungsrecht in Europa - Kernperspektiven am Ende des 20. Jahrhunderts, ZSR 2000, Beiheft 34, 13 (16); sowie Reichert-Facilides, Zur Kodifikation des deutschen internationalen Versicherungsvertragsrechts, IPRax 1990, 1 (3 f.).

13 Pearson, Opening Address, in: Reichert-Facilides / d'Oliveira (eds.), International Insurance Contract Law in the EC (1993)1 (5); vgl. auch R. M. Beckmann, Auswirkungen des EG-Rechts auf das Versicherungsvertragsrecht, ZEuP 1999, 809 (824 f.).

$14 \mathrm{Zu}$ diesem Ansatz Smulders / Glazener, Harmonization in the Field of Insurance Law through the Introduction of Community Rules of Conflict, CMLRev 29 (1992) 775.

15 Siehe die Zusammenstellung der maßgeblichen europarechtlichen Quellen bei Reichert-Facilides (Hrsg.), Aspekte des internationalen Versicherungsvertragsrechts im Europäischen Wirtschaftsraum (1994) 108 ff.; sowie den inhaltlichen Überblick bei Reichert-Facilides, Zur Kodifikation des deutschen internationalen Versicherungsvertragsrechts, IPRax 1990, 1 (4 ff.); sowie Migsch, Versicherungsvertragsrecht, in: Koppensteiner (Hrsg.), Österreichisches und europäisches Wirtschaftsprivatrecht, Teil $3 / 2$ (2000) 39 ff.; Kramer, Internationales Versicherungsvertragsrecht (1995) $132 \mathrm{ff}$.

16 Siehe die Auflistung der einschlägigen Richtlinien im Anhang IX/I. zum EWRAbkommen.

17 Näher zum Verhältnis des Kollisionsrechts in EG-Richtlinien im Allgemeinen zum EVÜ Czernich / Heiss-Helmberg, EVÜ (1999) Art. 20 Rn. 8 ff. 
EVÜ modifiziert. Dies gilt für Art. 6 Abs. 2 der Richtlinie über missbräuchliche Klauseln in Verbraucherverträgen ${ }^{18}$ sowie Art. 12 Abs. 2 der Richtlinie über den Fernabsatz von Finanzdienstleistungen an Verbraucher ${ }^{19}$. Sie enthalten Kollisionsnormen, die den Verbraucher im Binnenmarkt vor einer Rechtswahl schützen, die ein drittstaatliches Recht zur Anwendung beruft und damit potentiell den durch EG-Richtlinien gewährten Verbraucherschutz unterläuft. ${ }^{20}$ Diese Rechtswahlbeschränkungen finden auch bei Verbraucherversicherungen Anwendung.

\subsection{Defizite des heutigen Versicherungskollisionsrechts}

Die Ausführungen zur Sonderstellung des internationalen Versicherungsvertragsrechts im Rahmen des europäischen Kollisionsrechts haben es bereits angedeutet: Die europäische Gesetzgebung hat scharfe Kritik geerntet. ${ }^{21}$ Diese reicht von grundsätzlich-systematischer bis zu feiner Detailkritik. Die wichtigsten Punkte dieser Kritik stellen sich wie folgt dar:

\subsubsection{Rechtszersplitterung}

Erster Kritikpunkt am europäischen internationalen Versicherungsvertragsrecht ist die unnotwendige und zu größter Unübersichtlichkeit führende Zersplitterung der einschlägigen Rechtsquellen. ${ }^{22}$ Das EVÜ regelt das Kollisionsrecht für (alle) Rückversicherungsverträge sowie für Erstversicherungsverträge über Risiken, die nicht in einem Mitgliedstaat des euro-

18 Richtlinie 93 / 13 / EWG vom 5. 4. 1993 ABl. 1993 Nr. L 95 / 29.

19 Richtlinie 2002 / 65 / EG vom 23. 9. 2002 über den Fernabsatz von Finanzdienstleistungen an Verbraucher und zur Änderung der Richtlinie 90 / 619 / EWG des Rates und der Richtlinien 97/7/EG und 98/27/EG, ABl. 2002 Nr. L 271/16; zu ihrem kollisionsrechtlichen Gehalt Heiss, Die Richtlinie über den Fernabsatz von Finanzdienstleistungen an Verbraucher aus Sicht des IPR und des IZVR, IPRax 2003, 100.

20 Vgl. zu diesen Sonderkollisionsnormen z. B. Czernich / Heiss-Heiss, EVÜ (1999) Art. 5 Rn. 65 ff.; zur Umsetzung in Deutschland und Österreich Loacker, Der Verbrauchervertrag im internationalen Privatrecht (2006) $163 \mathrm{ff}$.

21 Vgl. nur - m. w. N. - Staudinger, Anknüpfung von Gerichtsstandsvereinbarungen und Versicherungsverträgen, in: Leible (Hrsg.), Das Grünbuch zum Internationalen Vertragsrecht (2004) 37 (46 f.).

22 Anschaulich dargestellt von Wandt, Internationales Privatrecht der Versicherungsverträge, in: Reichert-Facilides / Schnyder (Hrsg.), Versicherungsrecht in Europa - Kernperspektiven am Ende des 20. Jahrhunderts, ZSR 2000, Beiheft 34, 85 (87 f.); jüngst wiederum kritisch Wieser, Auf dem Weg zur Europapolizze?, VR 2006, 53 (54); vgl. den Versuch, die Rechtszersplitterung im Rahmen der nationalen Umsetzungsgesetzgebung zu beseitigen bzw. zurück zu drängen, bei Reichert-Facilides, Gesetzgebungsvorschlag, in: Reichert-Facilides (Hrsg.), Aspekte des internationalen Versicherungsvertragsrechts im Europäischen Wirtschaftsraum (1994) 75 (83); sowie ders., Zur Kodifikation des deutschen internationalen Versicherungsvertragsrechts, IPRax 1990, 1 (9); aus der Sicht deutschen Rechts ferner Gruber, Internationales Versicherungsvertragsrecht (1999) $305 \mathrm{~m}$. w. N. 
päischen Wirtschaftsraums belegen sind. Die genannten Richtlinien regeln demgegenüber Versicherungsverträge über Risiken, die in einem Mitgliedstaat des EWR belegen sind; dies allerdings nur dann, wenn auch der Versicherer in einem Mitgliedstaat des EWR zumindest niedergelassen ist. Zu diesen beiden Regelungsbereichen tritt daher, vom europäischen Gesetzgeber wohl ungewollt und übersehen, ein dritter, europarechtlich ungeregelter Bereich. Es handelt sich um Versicherungsverträge über Risiken, die in einem Mitgliedstaat des EWR belegen sind, jedoch nicht bei einem Versicherer mit Niederlassung in einem Mitgliedstaat in Deckung gegeben werden. Dieser letzte Bereich obliegt somit der autonomen Gesetzgebung der Mitgliedstaaten. ${ }^{23}$ Diese waren gut beraten, die Lücke systemkonform im Sinne des Richtlinienrechts zu schließen. Die auf europäischer Ebene herrschende Rechtszersplitterung garantierte dieses Vorgehen aber nicht.

\subsubsection{Wertungswidersprüche}

Die differenzierten Quellen des europäischen Versicherungskollisionsrechts weichen inhaltlich ganz grundsätzlich voneinander ab. ${ }^{24}$ Das EVÜ erkennt, auch für die von ihm umfassten Versicherungsverträge, den Grundsatz der Rechtswahlfreiheit an. ${ }^{25}$ Haben die Parteien keine Rechtswahl getroffen, so beruft Art. 4 EVÜ im Rahmen der objektiven Anknüpfung das Recht des Staates zur Anwendung, in dem der Versicherer niedergelassen ist. ${ }^{26}$ Nur bei Verbraucherversicherungen beschränkt Art. 5 EVÜ die Wirkungen einer Rechtswahl ${ }^{27}$ und sieht bei Fehlen einer solchen eine Anknüpfung an den gewöhnlichen Aufenthalt des Verbrauchers vor. ${ }^{28}$ Ergänzt wird diese Verbraucherkollisionsnorm noch durch die genannten

23 Aus deutscher Sicht zu dieser Dreiteilung E. Lorenz, Das auf grenzüberschreitende Lebensversicherungsverträge anwendbare Recht - eine Übersicht über die kollisionsrechtlichen Rechtsgrundlagen, ZVersWiss 1991, 121 (124 f.).

$24 \mathrm{Vgl}$. den Versuch, die Wertvorstellungen des EVÜ mit jenen des Richtlinienrechts zu verschmelzen bei Reichert-Faclides, Gesetzgebungsvorschlag, in: ReichertFacilides (Hrsg.), Aspekte des internationalen Versicherungsvertragsrechts im Europäischen Wirtschaftsraum (1994) 75 (83).

25 Art. 3 EVÜ; zur grundsätzlichen Bedeutung der Rechtswahlfreiheit im System des EVÜ Czernich / Heiss-Heiss, EVÜ (1999) Art. 3 Rn. 1 ff. m. w. N.; aus dem Blickwinkel des Versicherungskollisionsrechts Morse, Party Autonomy in International Insurance Contract Law, in: Reichert-Facilides / d'Oliveira (eds.), International Insurance Contract Law in the EC (1993) 23 (26).

26 Vgl. nur Wandt, Internationales Privatrecht der Versicherungsverträge, in: Reichert-Facilides / Schnyder (Hrsg.), Versicherungsrecht in Europa - Kernperspektiven am Ende des 20. Jahrhunderts, ZSR 2000, Beiheft 34, 85 (89).

27 Wandt, Internationales Privatrecht der Versicherungsverträge, in: Reichert-Facilides / Schnyder (Hrsg.), Versicherungsrecht in Europa - Kernperspektiven am Ende des 20. Jahrhunderts, ZSR 2000, Beiheft 34, 85 (89).

28 Wandt, Internationales Privatrecht der Versicherungsverträge, in: Reichert-Facilides / Schnyder (Hrsg.), Versicherungsrecht in Europa - Kernperspektiven am Ende des 20. Jahrhunderts, ZSR 2000, Beiheft 34, 85 (89). 
Verbraucherkollisionsnormen des Richtlinienrechts. ${ }^{29}$ Genau gegenteilig stellt sich der Regelungsansatz des Richtlinienkollisionsrechts für Versicherungen dar. Weder für Nicht-Lebensversicherungen noch für Lebensversicherungen wird von einem Grundsatz der freien Rechtswahl ausgegangen. ${ }^{30}$ Vielmehr sehen die Richtlinien eine objektive Anknüpfung des Versicherungsvertrags vor, die nur im Rahmen von vornherein beschränkter Rechtswahloptionen durch Rechtswahl verdrängt werden kann. ${ }^{31}$ Im Rahmen objektiver Anknüpfung stellt das Richtlinienrecht auch im Bereich von Nicht-Verbraucherverträgen auf die Belegenheit des Risikos (Nicht-Lebensversicherung) bzw. den Mitgliedstaat der Verpflichtung (Lebensversicherung) und damit in der ganz überwiegenden Zahl der Fälle auf den gewöhnlichen Aufenthalt bzw. die Niederlassung des Versicherungsnehmers ab. ${ }^{32}$ Es wird deutlich: Gegenläufiger hätten die Kollisionsrechtsregime für die vom EVÜ und vom Richtlinienrecht umfassten Versicherungsverträge kaum formuliert werden können.

\subsubsection{Unvollständigkeit der Rechtsharmonisierung}

Eine Harmonisierung des Kollisionsrechts erstrebt Planungssicherheit für die Vertragsparteien, jedenfalls was das anwendbare Recht anbelangt. Dies kann indessen nur dann vollständig gelingen, wenn die einschlägigen Richtlinien das internationale Versicherungsvertragsrecht der Mitgliedstaaten einander hinreichend näher bringen. Den Richtlinien zum Versicherungsrecht gelingt dies nur unzulänglich. An mehreren Stellen überlassen sie die Entscheidung über eine Rechtswahlmöglichkeit der Parteien bzw. über die Art und Weise einer objektiven Anknüpfung dem nationalen Gesetzgeber. Dies gilt etwa für Art. 7 Abs. 1 lit. a S 2, lit. d, lit. i und Art. 8 Abs. 4 lit. c der Zweiten Richtlinie Nicht-Lebensversicherung ${ }^{33}$. Dasselbe

29 Vgl. Kronke / Melis / Schnyder-Heiss / Schnyder, HdB Internationales Wirtschaftsrecht (2004) 175.

30 Einen solchen gibt es nur für Großrisikoversicherungen; näher hierzu Schnyder, Parteiautonomie im europäischen Versicherungskollisionsrecht, in: Reichert-Facilides (Hrsg.), Aspekte des internationalen Versicherungsvertragsrechts im Europäischen Wirtschaftsraum (1994) 49 (54 ff.); Morse, Party Autonomy in International Insurance Contract Law, in: Reichert-Facilides / d'Oliveira (eds.), International Insurance Contract Law in the EC (1993) 23 (37); R. M. Beckmann, Auswirkungen des EG-Rechts auf das Versicherungsvertragsrecht, ZEuP 1999, 809 (825).

31 Vgl. Morse, Party Autonomy in International Insurance Contract Law, in: Reichert-Facilides / d'Oliveira (eds.), International Insurance Contract Law in the EC (1993) 23 (39 und 47).

32 Vgl. Wandt, Internationales Privatrecht der Versicherungsverträge, in: ReichertFacilides /Schnyder (Hrsg.), Versicherungsrecht in Europa - Kernperspektiven am Ende des 20. Jahrhunderts, ZSR 2000, Beiheft 34, 85 (93 und 95).

33 Zweite Richtlinie 88 / 357 / EWG des Rates vom 22. Juni 1988 zur Koordinierung der Rechts- und Verwaltungsvorschriften für die Direktversicherung (mit Ausnahme der Lebensversicherung) und zur Erleichterung der tatsächlichen Ausübung des 
gilt für Art. 32 Abs. $1 \mathrm{~S}$ 2, Abs. 3 Unterabs. 2 und Abs. 4 Unterabs. 2 Richtlinie Lebensversicherung ${ }^{34}$. Es ist daher fraglich, ob der Grad der Rechtsharmonisierung für die Verwirklichung des Binnenmarkts ausreicht.

\subsubsection{Legistische Mängel}

In der Literatur werden auch erhebliche legistische Mängel des Richtlinienrechts moniert. Schon sein Regelungsaufbau verhindert, dass ein unbefangener Leser den Inhalt der Vorschriften unmittelbar nachvollziehen kann. Die Formulierung, Komplexität und Differenziertheit der Regelungen steigern den Grad an Unverständlichkeit. ${ }^{35}$ Nationale Umsetzungsgesetzgeber und insbesondere nationale Rechtsanwender waren und sind daher vor eine schwierige Aufgabe gestellt. Die Schwierigkeiten belasten letzten Endes die Vertragsparteien: Versicherungsverträge sind zumeist auf Dauer angelegte Risikogeschäfte. Die Parteien bedürfen daher rechtlicher Rahmenbedingungen, die ihnen Planungssicherheit gewähren. Diese Aufgabe kann das geltende Richtlinienkollisionsrecht für Versicherungsverträge nicht ausreichend erfüllen.

\subsubsection{Mangelnde Wirkkraft}

Die Rechtswissenschaft hat schon vor Erlass der einschlägigen Richtlinien darauf hingewiesen, dass die Kollisionsrechtsharmonisierung den Versicherungsbinnenmarkt im Massengeschäft nicht verwirklichen kann. ${ }^{36}$ Insbesondere würde es auf der Grundlage dieses Kollisionsrechtsregimes kaum zu mehr grenzüberschreitenden Abschlüssen von Versicherungsverträgen im Massengeschäft kommen. Diese Prognose blieb von der Kommission unbeachtet, hat sich aber bewahrheitet. EWR-Versicherer, die außerhalb des Staates ihrer Niederlassung belegene Massenrisiken versichern wollen, gründen regelmäßig im Zielstaat der Dienstleistung eine Tochtergesellschaft oder aber eine Niederlassung. Das im Dienstleistungsverkehr erbrachte, grenzüberschreitende Versicherungsgeschäft ist demgegenüber von statistisch verschwindend kleinem Ausmaß. ${ }^{37}$

freien Dienstleistungsverkehrs sowie zur Änderung der Richtlinie 73/239/EWG, ABl. 1988 Nr. L $172 / 1$.

34 Richtlinie 2002 / 83 / EG des EP und des Rates vom 5. 11.2002 über Lebensversicherungen (Richtlinie Lebensversicherung), ABl. 2002 Nr. L 345 / 1.

35 Siehe z. B. Reichert-Facilides, Synopsis of the Colloquy and Prospects for International Insurance Contract Legislation within the EC, in: Reichert-Facilides / d'Oliveira (eds.), International Insurance Contract Law in the EC (1993) 191 (196).

36 So ganz allgemein bereits W.-H. Roth, Internationales Versicherungsvertragsrecht (1985) $744 \mathrm{f}$.

37 Vgl. z. B. die Stellungnahme des Europäischen Wirtschafts- und Sozialausschusses zum Thema "Europäischer Versicherungsvertrag“ vom 15.12. 2004, ABl. 2005 Nr. C $157 / 1$, Pkt. 4.2.1.1; zu dieser Stellungnahme Heiss, Europäischer Ver- 
Dieser letzte Kritikpunkt des Richtlinienkollisionsrechts bedarf freilich einer Relativierung. Zu kritisieren ist nämlich nur, dass die Kommission Warnungen der Wissenschaft in den Wind geschrieben hat, eine Kollisionsrechtsharmonisierung reiche für die Binnenmarktverwirklichung nicht aus. Jedoch hätte auch eine Harmonisierung des internationalen Versicherungsvertragsrechts, die allen unter 1.2.2 bis 1.2.4 vorgebrachten Kritikpunkten gerecht würde, grenzüberschreitende Versicherungsdienstleistungen nur erleichtern, den Binnenmarkt letztlich aber nicht vollständig verwirklichen können. ${ }^{38}$ Hierfür ist eine Vereinheitlichung des materiellen Vertragsrechts der Massenrisikoversicherungen erforderlich. ${ }^{39}$

\subsubsection{Zwischenergebnis: Warten auf eine umfassende Kollisionsrechtsharmonisierung nach Art. 61 lit. c i.V.m. Art. 65 lit. b EGV}

Bei dem fast durchweg negativen Befund zur Qualität des geltenden europäischen Versicherungskollisionsrechts blieb in der Vergangenheit nur die Hoffnung auf eine Reform. Diese Hoffnung wurde gestärkt, als die EG durch Einfügung der neuen Kompetenznormen der Art. 61 lit. c i.V.m. Art. 65 EGV eine ausdrückliche Kompetenz zur sekundärrechtlichen Angleichung des Kollisionsrechts erhielt. ${ }^{40}$ Wenngleich nämlich eine Änderung des EVÜ nur sehr schwer denkbar war, weil sie die Zustimmung und die Ratifikation durch alle Vertragsstaaten erfordert hätte ${ }^{41}$, war und ist ein „Umgießen“ des EVÜ in einen Sekundärrechtsakt (z. B. eine EG-Verordnung) weit eher vorstellbar. ${ }^{42}$

sicherungsvertrag - Initiativstellungnahme des Europäischen Wirtschafts- und Sozialausschusses verabschiedet, VersR 2005, 1.

38 Auch eine Liberalisierung des internationalen Versicherungsvertragsrechts durch Einräumung unbeschränkter Rechtswahlfreiheit würde den Binnenmarkt nicht verwirklichen; kritisch zu diesem Ansatz Basedow, Die Gesetzgebung zum Versicherungsvertrag zwischen europäischer Integration und Verbraucherpolitik, in: Reichert-Facilides / Schnyder (Hrsg.), Versicherungsrecht in Europa - Kernperspektiven am Ende des 20. Jahrhunderts, ZSR 2000, Beiheft 34, 13 (20 f.).

39 Sie erscheint auch möglich: vgl. Rühl, Common Law, Civil Law, and the Single European Market for Insurances, ICLQ 55 (2006) 879, die dartut, dass auch die „Kluft" zwischen englischem und kontinentaleuropäischem Versicherungsvertragsrecht überbrückt werden kann.

40 Hierzu Dohrn, Die Kompetenzen der EG im Internationalen Privatrecht (2004).

41 Die Hoffnung auf eine Revision des EVÜ bei Gruber, Internationales Versicherungsvertragsrecht (1999) 311 hat sich bislang leider nicht bestätigt.

42 Hierzu näher Staudinger, Anknüpfung von Gerichtsstandsvereinbarungen und Versicherungsverträgen, in: Leible (Hrsg.), Das Grünbuch zum Internationalen Vertragsrecht (2004) 37 (50 ff.). 


\title{
2. Reformvorschläge von Kommission, Parlament und Rat
}

\author{
2.1 Vorschlag der Kommission für eine Verordnung \\ des EP und des Rates über das auf vertragliche Schuldverhältnisse \\ anzuwendende Recht (Rom I-Vorschlag) ${ }^{43}$ aus 2005
}

\subsubsection{Ausklammerung des Binnenmarktkollisionsrechts für Versicherungsverträge}

Die beschriebene Hoffnung wurde auf erstes Hinsehen durch den von der Kommission vorgelegten Rom-I-Vorschlag genährt. Darin fehlt eine Parallelbestimmung zu Art. 1 Abs. 3 EVÜ, der Erstversicherungsverträge über Risiken, die in einem EG-Mitgliedstaat belegen sind, vom Anwendungsbereich ausnehmen würde. Wer im Rom-I-Vorschlag allerdings weiter liest, wird doppelt enttäuscht. Artikel 22 lit. a stellt nämlich in Verbindung mit Anhang I klar, dass das Kollisionsrecht der Versicherungsrichtlinien vom Rom-I-Vorschlag unberührt bleiben soll. Rechtszersplitterung, Wertungswidersprüche, die Unvollständigkeit der Harmonisierung und auch die legistischen Mängel des Richtlinienrechts bleiben also erhalten. ${ }^{44} \mathrm{Mehr}$ noch: Zumal nicht alle Versicherungsverträge über Risiken, die in einem EWR-Mitgliedstaat belegen sind, vom Anwendungsbereich des Vorschlags ausgenommen werden, sondern nur jene, die von der Richtlinienregelung umfasst sind, schließt der Rom-I-Vorschlag nunmehr neben den Versicherungsverträgen über in Drittstaaten belegene Risiken auch solche Versicherungsverträge ein, die zwar im EWR belegene Risiken decken, jedoch mit Versicherern aus Drittstaaten geschlossen werden. Damit wird der nach geltendem Europarecht dem mitgliedstaatlichen Gesetzgeber überlassene Regelungsbereich vom Rom-I-Vorschlag abgedeckt. Zumal nationale Gesetzgeber diesen Bereich sinnvoll durch eine autonome Erstreckung des Richtlinienrechts regelten, würde der Rom-I-Vorschlag eine entsprechende Anpassung der Umsetzungsgesetze in den Mitgliedstaaten erfordern. ${ }^{45} \mathrm{Mehr}$ noch: Er würde den Wertungswiderspruch zwischen dem Regime des EVÜ und dem Richtlinienrecht in den Binnenmarkt hineintragen, indem jedenfalls Versicherungsverträge über im EWR belegene Risiken einem jeweils ganz anders gearteten Kollisionsrechtsregime unterstehen würden, je nachdem, ob der Versicherer im EWR niedergelassen ist oder nicht. ${ }^{46}$ Im Ergeb-

43 KOM (2005) 650 endgültig.

44 Heiss, Das Kollisionsrecht der Versicherungsverträge nach Rom I und II, VersR 2006, 185 (185); Staudinger, Internationales Versicherungsvertragsrecht, in: Ferrari / Leible (Hrsg.), Ein neues Internationales Vertragsrecht für Europa (2007) 225 (233); Wieser, Auf dem Weg zur Europapolizze?, VR 2006, 53 (57 f.).

45 Heiss, Das Kollisionsrecht der Versicherungsverträge nach Rom I und II, VersR 2006, 185 (186 f.).

46 Heiss, Das Kollisionsrecht der Versicherungsverträge nach Rom I und II, VersR 2006, 185 (187). 
nis folgt: Der Rom-I-Vorschlag enttäuscht die Hoffnung der Versicherungskollisionsrechtler und verschlechtert sogar den Ist-Zustand.

\subsubsection{Erste Schritte in Richtung eines „optionalen europäischen Vertragsrechts“}

Proponenten einer Verwirklichung des Binnenmarkts für Versicherungen schöpfen indessen an anderer Stelle des Rom-I-Vorschlags neue Hoffnung: Art. 3 Abs. 2 des Vorschlags lässt nämlich den Parteien neben einer Rechtswahl im engeren Sinne auch die Möglichkeit, ihrem Vertrag ,auf internationaler oder Gemeinschaftsebene anerkannte Grundsätze und Regeln des materiellen Vertragsrechts" zugrunde zu legen. ${ }^{47}$ Darüber hinaus behält Art. 22 lit. b Rom-I-Vorschlag ausdrücklich die Möglichkeit des EG-Gesetzgebers vor, in Zukunft neben der Rom-I-Verordnung auch ein optionales europäisches Vertragsrecht zu schaffen. ${ }^{48}$ Gerade in der versicherungsrechtlichen Literatur wurde ja vielfach hervorgehoben, dass die (international) zwingenden Regeln des Zuständigkeitsrechts, des Kollisionsrechts, aber auch des materiellen Rechts der Versicherungsverträge den grenzüberschreitenden Vertrieb von Europapolicen vereiteln. ${ }^{49}$ Nur eine materielle Rechtsvereinheitlichung, idealtypisch in Form eines optionalen Instruments, könne der Binnenmarktverwirklichung dienen. ${ }^{50}$ Gerne liest daher gerade der Versicherungsjurist die neuen Wahlmöglichkeiten des Rom-

47 Hierzu z. B. Jud, Neue Dimensionen privatautonomer Rechtswahl, JBl 2006, 695; Heiss, Die Vergemeinschaftung des internationalen Vertragsrechts durch „Rom I“ und ihre Auswirkungen auf das österreichische IPR, JBl 2006, 750 (758 f.); Leible, Rechtswahl, in: Ferrari/Leible (Hrsg.), Ein neues Internationales Vertragsrecht für Europa, 41 (46); kritisch Mankowski, Stillschweigende Rechtswahl und wählbares Recht, in: Leible (Hrsg.), Das Grünbuch zum Internationale Vertragsrecht (2004) 63 (90 ff.); ders., Der Vorschlag für die Rom I-Verordnung, IPRax 2004, 101; vorsichtig auch Magnus, Der Kommissionsvorschlag der Rom I Verordnung - Nachbesserungen sind noch nötig, EWS 2006, Heft 5, Die erste Seite.

48 Heiss, Die Vergemeinschaftung des internationalen Vertragsrechts durch „Rom I" und ihre Auswirkungen auf das österreichische IPR, JBl 2006, 750 (754 f.); näher zum optionalen Instrument Staudenmayer, Ein optionelles Instrument im Europäischen Vertragsrecht?, ZEuP 2003, 828; Basedow, Ein optionales Europäisches Vertragsgesetz - Opt-in, opt-out, wozu überhaupt? ZEuP 2004, 1; zu kollisionsrechtlichen Folgefragen Heiss / Downes, Non-Optional Elements in an Optional European Contract Law. Reflections from a Private International Law Perspective, ERPL 13 (2005), 693.

$49 \mathrm{Zu}$ einem optionalen Versicherungsvertragsrecht Basedow, Insurance Contract Law as Part of an Optional European Contract Act ERA-Forum (2003), 56; ders., Der Versicherungsbinnenmarkt und ein optionales europäisches Vertragsgesetz, in: FS E. Lorenz (2004) 93; Heiss, Mobilität und Versicherung, VersR 2006, 448 (453); Wieser, Auf dem Weg zur Europapolizze?, VR 2006, 53 (60 f.).

50 Demgegenüber kann der derzeit in Ausarbeitung befindliche Gemeinsame Referenzrahmen europäischen Vertragsrechts nur als ein Teilschritt zur Verwirklichung des Versicherungsbinnenmarktes angesehen werden; ausführlich hierzu Basedow, Der Gemeinsame Referenzrahmen und das Versicherungsvertragsrecht, ZEuP 2007, 280. 
I-Vorschlags. ${ }^{51}$ Freilich enttäuscht die Kommission den Versicherungsjuristen auch in diesem Punkt, weil das Richtlinienkollisionsrecht der Versicherungsverträge gemäß Art. 22 lit. a i.V.m. Anhang I Rom-I-Vorschlag unberührt bleiben soll. Dies hätte dann wohl auch zu bedeuten, dass Art. 3 Abs. 2 Rom-I-Vorschlag sowie Art. 22 lit. b Rom-I-Vorschlag (optionales Instrument) nicht für Versicherungsverträge im Binnenmarkt vorgeschlagen wurden. ${ }^{52}$

\subsection{Die Position des Europäischen Parlaments}

\subsubsection{Integration des Binnenmarktkollisionsrechts für Versicherungsverträge}

Zum Rom-I-Vorschlag der Kommission liegt bis dato noch keine endgültige Äußerung des Europäischen Parlaments, immerhin aber der Entwurf eines Berichts ${ }^{53}$ vom 22. 8. 2006 vor. Dieser enthält diverse Änderungsanträge zum Rom-I-Vorschlag und wurde am 7.12. $2006^{54}$, am 5.3. $2007^{55}$ und am 23. 5. $2007^{56}$ durch weitere Änderungsanträge von Mitgliedern des EP ergänzt. Was das internationale Versicherungsvertragsrecht angeht, so zeichnet sich eine erfreuliche Tendenz zu dessen Integration in den RomI-Vorschlag ab. Damit würden jedenfalls das Problem der Rechtszersplitterung, der damit einhergehenden, unsachlich differenzierenden Wertung und - durch die Schaffung von Einheitsrecht - auch das Problem der Unvollständigkeit der Rechtsharmonisierung beseitigt.

51 Vgl. z. B. Staudinger, Internationales Versicherungsvertragsrecht, in: Ferrari / Leible (Hrsg.), Ein neues Internationales Vertragsrecht für Europa (2007) 225 (230).

52 Näher hierzu Heiss, Das Kollisionsrecht der Versicherungsverträge nach Rom I und II, VersR 2006, 185 (186); ähnlich Basedow, Der Gemeinsame Referenzrahmen und das Versicherungsvertragsrecht, ZEuP 2007, 280 (287).

53 Entwurf eines Berichts über den Vorschlag für eine Verordnung des Europäischen Parlaments und des Rates über das auf vertragliche Schuldverhältnisse anzuwendende Recht (Rom I) (KOM(2005)0650-C6-0041/2005-2005 / 0261 (COD)), Berichterstatterin: Maria Berger, Änderungsanträge $1-31$ vom 22. 8. 2006, PE-374.427v01-00 (im Folgenden zitiert als: Entwurf eines Berichts I).

54 Entwurf eines Berichts über den Vorschlag für eine Verordnung des Europäischen Parlaments und des Rates über das auf vertragliche Schuldverhältnisse anzuwendende Recht (Rom I) (KOM(2005)0650-C6-0041 / 2005-2005 / 0261 (COD)), Berichterstatterin: Maria Berger, Änderungsanträge 32 - 85 vom 7. 12.2006, PE-382.371v01-00 (im Folgenden zitiert als: Entwurf eines Berichts II).

55 Entwurf eines Berichts über den Vorschlag für eine Verordnung des Europäischen Parlaments und des Rates über das auf vertragliche Schuldverhältnisse anzuwendende Recht (Rom I) (KOM(2005)0650-C6-0041/2005-2005 / 0261 (COD)), Berichterstatter: Cristian Dumitrescu, Änderungsanträge 86-96 vom 5. 3. 2007, PE-386.328v01-00 (im Folgenden zitiert als: Entwurf eines Berichts III).

56 Entwurf eines Berichts über den Vorschlag für eine Verordnung des Europäischen Parlaments und des Rates über das auf vertragliche Schuldverhältnisse anzuwendende Recht (Rom I) (KOM(2005)0650-C6-0041/2005-2005/0261 (COD)), Berichterstatter: Cristian Dumitrescu, Kompromissänderungsanträge 1-1 vom 23.5. 2007, PE-390.396v01-00 (im Folgenden zitiert als: Entwurf eines Berichts IV). 


\subsubsection{Versicherungsnehmer- oder Verbraucherschutz?}

2.2.2.1 Vorschlag 1:

Reduktion des Versicherungsnehmerschutzes auf den Verbraucherschutz

Im Entwurf eines Berichts über den Rom-I-Vorschlag war zunächst angedacht, den Versicherungsnehmerschutz auf den Schutz des privaten Endverbrauchers zu reduzieren. ${ }^{57}$ Auch in der Versicherungsbranche bedürften nämlich im Rechtswahlfalle nur Verbraucher des Schutzes. Unternehmer hätten demgegenüber hinreichende Geschäftserfahrungen, „um die Risiken einschätzen zu können, die sie bei einer Tätigkeit außerhalb der Rechtsordnung ihres Heimatlandes eingehen, bzw. um zu erkennen, wann sie rechtlichen Beistand benötigen. "58 Diese Begründung findet sich beinahe wortgleich in der Stellungnahme ${ }^{59}$ des Europäischen Wirtschafts- und Sozialausschusses (EWSA) zum Rom-I-Vorschlag vom 13. 9. 2006.

Die vorgeschlagene Zurücknahme des Versicherungsnehmerschutzes erscheint weder rechtssystematisch noch rechtspolitisch vertretbar. In rechtssystematischer Sicht stellt der Vorschlag zu allererst einen Bruch mit der EuGVVO dar, die im internationalen Zuständigkeitsrecht für Versicherungssachen und insbesondere im Kontext der freien Gerichtsstandswahl auch den gewerbetreibenden Versicherungsnehmer bis hin zur Grenze der Großrisikoversicherung schützt. ${ }^{60}$ Gleiches gilt im Vergleich zum materiellen Versicherungsvertragsrecht, zu vorderst zum deutschen VVG. Dieses schützt gemäß seinem $\S 187$ auch gewerblich tätige Versicherungsnehmer bis hin zur Grenze der Großrisikoversicherungen. Ähnlich ist die Rechtslage in den meisten europäischen Versicherungsvertragsgesetzen. ${ }^{61}$ Mit Recht wurde daher vorgeschlagen, im Zuge einer Harmonisierung des Versicherungsvertragsrechts, den Schutzstandard nicht auf private Endverbraucher zu reduzieren. Auch die Projektgruppe „Restatement of European Insurance Contract Law" folgt bei der Ausformulierung des gemeinsamen Referenzrahmens eines europäischen Versicherungsvertragsrechts (den „Principles of European Insurance Contract Law, PEICL“) diesem Ansatz. ${ }^{62}$ Die Systemwidrigkeit der vorgeschlagenen Reduktion des Versicherungsneh-

57 Siehe den Änderungsantrag 31 im Entwurf eines Berichts I.

58 Entwurf eines Berichts I, Begründung zu Änderungsantrag 31.

59 Stellungnahme vom 13. 9. 2006 zum Vorschlag für eine Verordnung des Europäischen Parlaments und des Rates über das auf vertragliche Schuldverhältnisse anzuwendende Recht (Rom I) (KOM(2005)0650-2005 / 0261 (COD)), INT / 307.

60 Siehe insbesondere Art. 13 Nr. 5 i.V.m. Art. 14 Nr. 5 EuGVVO.

61 Vgl. Basedow/Fock, Rechtsvergleich, in: Basedow/Fock (Hrsg.), Europäisches Versicherungsvertragsrecht I (2002) $11 \mathrm{ff}$.

62 Die Regelung wird in Art. 1:103 PEICL enthalten sein; zur Arbeit der Restatement Group www.restatement.info 
merschutzes auf den Verbraucherschutz indiziert bereits seine rechtspolitische Untragbarkeit. Unübersehbar streiten jedenfalls drei Argumente für einen Schutz auch von Unternehmern im Versicherungsrecht: Erstens das typischerweise vorliegende Machtgefälle, welches sich nicht nur auf den Vertragsschluss, sondern auch die Vertragsabwicklung und insbesondere im Leistungsfall auswirkt; zweitens die Komplexität von Versicherungsprodukten, aus der nicht zuletzt ein Informationsgefälle zwischen Versicherer und Versicherungsnehmer resultiert ${ }^{63}$; drittens die häufig existentielle Bedeutung, die das Produkt "Versicherungsschutz" nicht nur für private Endverbraucher, sondern gerade auch für klein- und mittelständische Unternehmen besitzt. Es ist daher zu begrüßen, dass dieser Vorschlag in späteren Änderungsanträgen zurückgenommen wurde.

\subsubsection{Vorschlag 2:}

Beibehaltung des Versicherungsnehmerschutzes

Demgegenüber will ein späterer Änderungsantrag Nr. 68 die Anknüpfung von Versicherungsverträgen in einem eigenen Artikel 5a der vorgeschlagenen Rom-I-Verordnung regeln. ${ }^{64}$ Diese Bestimmung schließt nicht nur ihrer Stellung nach nahtlos an Art. 5 Rom-I-Vorschlag an, sondern lehnt sich auch inhaltlich daran an. So knüpft Absatz 1 an den gewöhnlichen Aufenthalt des Versicherungsnehmers an. Der Schutz des vorgeschlagenen Art. 5a beschränkt sich somit nicht auf private Endverbraucher, sondern erstreckt sich auch auf Unternehmer. Die Grenze markiert Art. 5a Abs. 4, der Rückversicherungen und Versicherungsverträge über Großrisiken vom Anwendungsbereich der Vorschrift ausnimmt.

Dieser neue Änderungsantrag ist im Grundsatz zu begrüßen. Die systematische Eingliederung des internationalen Versicherungsvertragsrechts in Art. 5a Rom-I-Vorschlag erscheint gelungen. Der Schutz (auch) von Unternehmern überzeugt rechtspolitisch und bewegt sich im System des internationalen und nationalen Versicherungsrechts. Stimmig ist auch die Zurücknahme des Versicherungsnehmerschutzes bei Rück- und Großrisikoversicherungen.

63 Auf dieses Informationsgefälle stellen Basedow/Fock, Rechtsvergleich, in: Basedow / Fock (Hrsg.), Europäisches Versicherungsvertragsrecht I (2002) 14 besonders ab.

64 Siehe den Änderungsantrag 68 im Entwurf eines Berichts II; unklar bleibt dessen Verhältnis zum Änderungsantrag 48 im Entwurf eines Berichts II, wonach sich die objektive Anknüpfung von Versicherungsverträgen nach Art. 4 Abs. 1 lit b), ha) einer Rom-I-Verordnung an der Belegenheit des Risikos ausrichten soll; vermutlich macht der Änderungsantrag 68 den Änderungsantrag 48 gegenstandslos. Möglich wäre auch, dass Änderungsantrag 48 für Versicherungen gedacht ist, die nicht in den Regelungsbereich des in Änderungsantrag 68 vorgeschlagenen Art. 5a einer RomI-Verordnung fallen (Rück- und Großrisikoversicherungen). 


\subsubsection{Anknüpfung von Rückversicherungen}

Der neue Änderungsantrag Nr. 68 nimmt Rückversicherungsverträge vom Geltungsbereich des vorgeschlagenen Art. 5a Rom-I-Vorschlag aus. Dahinter steht nicht nur der Gedanke, dass Erstversicherer beim Abschluss von Rückversicherungsverträgen keines (zwingenden) kollisionsrechtlichen Versicherungsnehmerschutzes bedürfen, sondern auch die Beobachtung, dass auch die objektive Anknüpfung von Rückversicherungsverträgen traditionell anderen Gesichtspunkten folgt, als dies bei Erstversicherungen der Fall ist. ${ }^{65}$ Es ist daher konsequent, dass nicht nur der zwingende Charakter des Art. 5a Rom-I-Vorschlag zurückgenommen, sondern die Vorschrift auf Rückversicherungsverträge überhaupt keine Anwendung finden soll.

\subsubsection{Anknüpfung von Großrisikoversicherungen}

Der neue Änderungsantrag Nr. 68 nimmt auch Großrisikoversicherungen vom Anwendungsbereich des vorgeschlagenen Art. 5a Rom-I-Verordnung aus. Hierfür kann der mangelnde Schutzbedarf von Großkunden ins Treffen geführt werden. Allerdings rechtfertigt der fehlende Schutzbedarf nur die Rücknahme der zwingenden Natur des vorgeschlagenen Art. 5a einer RomI-Verordnung, nicht aber die Ausnahme von Großrisikoversicherungen schlechthin. Jedenfalls greifen die für die abweichende objektive Anknüpfung der Rückversicherung ins Treffen geführten Argumente im Falle der Großrisikenversicherung nicht. ${ }^{66}$ Der Unterschied zu den Massenrisiken sollte daher lediglich darin bestehen, dass im Falle der Großrisikoversicherung von der objektiven Anknüpfung durch eine freie Rechtswahl abgewichen werden kann.

Auch systematische Aspekte deuten in dieselbe Richtung: Nach der EuGVVO unterliegen auch Versicherungsverträge über Großrisiken dem Regime der Art. 8 ff. Für sie besteht lediglich nach Art. 13 Nr. 5 (insbesondere i.V.m. Art. 14 Nr. 5) EuGVVO die freie Gerichtsstandswahl. Auch die schon angesprochenen PEICL werden diesen Ansatz verfolgen. ${ }^{67}$ Dasselbe gilt übrigens auch für die Behandlung der Großrisikoversicherungen nach deutschem Versicherungsvertragsrecht. Dieses findet auch auf Großrisikoversicherungen Anwendung, § 187 VVG nimmt für diesen Bereich nur den zwingenden Charakter der Vorschriften des VVG zurück.

Auch der im neuen Änderungsantrag Nr. 68 vorgeschlagene Art. 5a Abs. 2 Rom-I-Verordnung spricht für eine Erstreckung des Anwendungsbereichs

65 Siehe nur W.-H. Roth, Internationales Versicherungsvertragsrecht (1985) 580 ff.; zur Debatte um die objektive Anknüpfung der Rückversicherung Mankowski, Internationales Rückversicherungsvertragsrecht, VersR 2002, 1177.

66 Siehe W.-H. Roth, Internationales Versicherungsvertragsrecht (1985) 584 f. und $587 \mathrm{ff}$.

67 Die Regelung wird in Art. 1:103 PEICL enthalten sein. 
von Art. 5a auf Großrisikoversicherungen. Art. 5a Abs. 2 unterwirft Pflichtversicherungsverträge dem Recht des Staates, der die Versicherungspflicht vorschreibt. Im Sinne der Schutzzwecke des Pflichtversicherungsrechts (insbesondere: Schutz des Geschädigten) sollte dasselbe auch dann gelten, wenn die Pflichtversicherung ein Großrisiko betrifft. ${ }^{68}$ Dieser Abs. 2 fände aber keine Anwendung, würde man Großrisikoversicherungen vom Anwendungsbereich des vorgeschlagenen Art. 5a ausnehmen.

$\mathrm{Zu}$ hinterfragen ist auch die vorgesehene Definition des Großrisikos. Der vorgeschlagene Art. 5a verweist hierfür auf die bestehende Definition im Richtlinienrecht. Das stößt auf mindestens zwei Bedenken: Zum einen wird mit der Neukodifizierung des internationalen Versicherungsvertragsrechts im Rahmen der Rom-I-Verordnung auch die Definition des Großrisikos in den Richtlinien für die Zwecke des Kollisionsrechts obsolet. Sie sollte daher in die Rom-I-Verordnung übernommen werden. Zum anderen enthält auch die EuGVVO einen Verweis auf das Richtlinienrecht. ${ }^{69}$ Sie räumt aber auch noch eine weitergehende Gerichtsstandswahlmöglichkeit bei speziellen Risiken ein. ${ }^{70}$ Ein Gleichlauf der Parteiautonomie im Zuständigkeits- und im Kollisionsrecht kann daher mit einem Verweis auf das Richtlinienrecht nicht erreicht werden. Besser wäre im Sinne des Gleichlaufs ein Verweis auf die EuGVVO. Dieser Verweis sollte mit einer Neuformulierung der Art. 13 Abs. 5 i.V.m. 14 EuGVVO, die Großrisiken einer stimmigeren Definition zuführt, Hand in Hand gehen. ${ }^{71}$

\subsubsection{Rechtswahlbeschränkung}

Als missglückt ist im vorgeschlagenen Art. 5a die Regelung der Rechtswahl zu bezeichnen. Art. 5a Abs. 3 eröffnet nur eine einzige Rechtswahlmöglichkeit. Ihr zufolge können die Vertragsparteien, wenn und solange der Vertrag keinen Verbrauchervertrag nach Art. 5 Abs. 1 darstellt, den Vertrag dem Recht des Landes unterstellen, in dem das Risiko belegen ist. ${ }^{72}$

Dieser Vorschlag ist zunächst technisch mangelhaft, weil der Begriff der Risikobelegenheit in Art. 5a Abs. 3 nicht definiert wird. Man mag hier auf die Definition in den Versicherungsrichtlinien zurückgreifen, doch beseitigt

68 So kann z. B. eine Haftpflichtversicherung eine Großrisiko- und zugleich eine Pflichtversicherung sein.

69 Art. 13 Nr. 5 i.V.m. Art. 14 Nr. 5 EuGVVO.

70 Art. 13 Nr. 5 i.V.m. Art. 14 Nr. 1 - 4 EuGVVO; hierzu Hub, Internationale Zuständigkeit in Versicherungssachen nach der VO 44 / 01 / EG (EuGVVO) (2005) 175.

71 Zur missglückten Regelung von Art. 13 Nr. 5 i.V. m. Art. 14 EuGVVO Hub, Internationale Zuständigkeit in Versicherungssachen nach der VO 44/01/EG (EuGVVO) (2005) 176.

72 Insofern dem geltenden Recht, Art. 7 Abs. 1 lit b) Zweiter RL Nicht-Lebensversicherung (ABl. 1988 Nr. L 172 / 1), ähnlich. Dort wird die Rechtswahlmöglichkeit allerdings auch im Verbrauchergeschäft unbeschränkt gewährt. 
das nicht alle Mängel: Zum einen erfährt der unbefangene Leser des Art. 5a nicht, dass eine solche Definition im Richtlinienrecht existiert. Zum anderen wäre es wünschenswert, wenn ein künftiger Art. 5a Rom-I-Verordnung nicht nur die eigentlichen Verweisungsnormen des Richtlinienrechts, sondern eben auch die „Hilfsnormen“, also die Definition der Risikobelegenheit beseitigen würde. ${ }^{73}$

Rechtspolitisch gesehen ist die vorgesehene Rechtswahlmöglichkeit $\mathrm{zu}$ eng gezogen. Das zeigt zunächst ein Vergleich mit Art. 5 (Verbraucherverträge). Gemäß Art. 5 Abs. 2 wird der Verbraucherschutz zurückgenommen und die Parteien können daher das auf den Vertrag anwendbare Recht nach Art. 3 frei wählen, wenn der Unternehmer im Verbraucherland nicht in irgendeiner Weise geschäftlich tätig geworden ist. Mit anderen Worten: Der aktiv grenzüberschreitend nachfragende Verbraucher genießt keinen kollisionsrechtlichen Verbraucherschutz und ist der freien Rechtswahl ausgesetzt. Vieles spricht dafür, eine solche Rechtswahl auch in Art. 5a für Versicherungsverträge zuzulassen. Schon wegen der (passiven) Dienstleistungsfreiheit, die ja auch der Kunde genießt, sollte es dem Versicherungsnehmer ermöglicht werden, grenzüberschreitend ausländische Versicherungsprodukte nachzufragen. ${ }^{74}$ Dies setzt allerdings eine Rechtswahlmöglichkeit zugunsten des Rechts des Staates voraus, in dem er den Versicherungsschutz nachfragt. Diese Möglichkeit spielt insbesondere für jene Versicherungsnehmer eine besondere Rolle, die sich gewöhnlich in einem Staat mit einem unterentwickelten privaten Versicherungssystem aufhalten. So ist es gut vorstellbar, dass die in Transformationsländern niedergelassenen Versicherer gewisse, etwa hoch spezialisierte Versicherungsprodukte nicht anbieten. In einem solchen Fall werden Versicherungsnehmer aktiv grenzüberschreitend Versicherungsschutz im Ausland nachfragen. Erfolgreich wird eine solche Nachfrage allerdings nur sein, wenn auch eine Rechtswahl zugunsten des Rechts am Sitz des Versicherers offensteht. Schutzgesichtspunkte widersprechen einer solchen Rechtswahl nicht: Jener Versicherungsnehmer, der sich aus eigenem Antrieb bei der Suche nach Versicherungsschutz an ausländische Bieter wendet, benötigt ebenso wenig Schutz, wie ein Verbraucher, der seinen Konsum aus eigenem Antrieb im Ausland befriedigt. ${ }^{75}$ Und allemal ist es besser, den Versicherungsnehmern Zugang zu

73 Hierzu noch unten 3.6.

74 Vgl. auch den Ansatz des Art. 28 Dritte RL Nicht-Lebensversicherung (ABl. 1992 Nr. L 228 / 1); zu dessen kollisionsrechtlicher Bedeutung nur W.-H. Roth, Dienstleistungsfreiheit und Allgemeininteresse im europäischen internationalen Versicherungsvertragsrecht, in: Reichert-Facilides (Hrsg.), Aspekte des internationalen Versicherungsvertragsrechts im Europäischen Wirtschaftsraum (1994) 1 (26 f.).

75 So auch W.-H. Roth, Dienstleistungsfreiheit und Allgemeininteresse im europäischen internationalen Versicherungsvertragsrecht, in: Reichert-Facilides (Hrsg.), Aspekte des internationalen Versicherungsvertragsrechts im Europäischen Wirtschaftsraum (1994) 1 (26). 
ausländischen Versicherungsmärkten zu verschaffen, als ihnen einen solchen selbst bei aktiver Nachfrage im Ausland zu verweigern.

Auch ein Vergleich mit dem geltenden Richtlinienrecht spricht für eine Erweiterung der Rechtswahl. Obwohl auch dieses keine freie Rechtswahl vorsieht, kennt es doch weitergehende Rechtswahlmöglichkeiten als der im Änderungsantrag vorgeschlagene Art. 5a Abs. $3 .^{76}$ Es besteht jedoch kein Grund, die Rechtswahlmöglichkeiten noch enger zu ziehen als nach geltendem Recht. Insgesamt zeigt sich: Die in Art. 5a Abs. 3 vorgesehene Rechtswahlmöglichkeit ist viel zu eng geraten.

\subsubsection{Pflichtversicherung}

Stimmig ist demgegenüber die Regelung der Pflichtversicherung in Art. 5a Abs. 2, wonach diese dem Recht des Staates unterliegt, der die Versicherungspflicht vorschreibt. Zwar könnte man hier an differenzierte Lösungen denken, wonach z. B. grundsätzlich das Recht des gewöhnlichen Aufenthalts des Versicherungsnehmers zur Anwendung kommt, welches von den zwingenden Pflichtversicherungsvorschriften des Staates, der die Pflicht vorschreibt, allerdings partiell wieder verdrängt wird ${ }^{77}$ Eine derart differenzierende Lösung führte jedoch zu einem unnötigen law mix, der in der Rechtsanwendung große praktische Probleme erzeugen kann. Die Einfachheit der Regelung spricht daher für den vorgeschlagenen Art. 5a Abs. 2.

\subsection{Die Vorschläge der Ratspräsidentschaft}

\subsubsection{Integration des Binnenmarktkollisionsrechts für Versicherungsverträge}

Ein Papier der Ratspräsidentschaft zum Rom-I-Vorschlag vom 2. 3. 2007 i. d. F. 4. 5. $2007^{78}$ hat zum Teil erheblich in den Kommissionsvorschlag eingegriffen. Das Papier verfolgt dabei den auch vom Europäischen Parlament vertretenen Ansatz, das Kollisionsrecht der Versicherungsverträge in einen Art. 5a und somit vollständig in die Rom-I-Verordnung zu integrieren. Insofern ist das Papier der Ratspräsidentschaft zu begrüßen.

\footnotetext{
$76 \mathrm{Zu}$ den Optionen nach dem Richtlinienrecht im Einzelnen Morse, Party Autonomy in International Insurance Contract Law, in: Reichert-Facilides/d'Oliveira (eds.), International Insurance Contract Law in the EC (1993) 23 (39 ff. und $47 \mathrm{ff}$.).

77 Vgl. den Ansatz des $§ 11$ des österreichischen IVVG.

78 Vermerk des Vorsitzes für den Ausschuss für Zivilrecht vom 2. 3. 2007, Dokument Nr. $6935 / 07$ i. d. F. Dokument Nr. 8935/1/07 (im Folgenden zitiert als: Papier der Ratspräsidentschaft).
} 


\subsubsection{Integration der Rückversicherung}

Der im Papier der Ratspräsidentschaft vorgeschlagene Art. 5a umfasst auch eine Regelung der Rückversicherung in Art. 5a Abs. 1. Demnach sollen Rückversicherungsverträge der freien Rechtswahl nach Art. 3 und, mangels einer Rechtswahl, dem Recht am gewöhnlichen Aufenthalt des Rückversicherers unterstehen. Diese Regelung kann kaum schaden, weil Rückversicherungsverträge regelmäßig Rechtswahlklauseln enthalten, die das Problem ohnehin im Wege der Parteiautonomie lösen. Man mag allenfalls darüber streiten, ob die objektive Anknüpfung an den gewöhnlichen Aufenthalt des Rückversicherers dem Kriterium der engsten Verbindung gerecht wird. ${ }^{79}$ Insofern wäre es wohl tatsächlich besser, Rückversicherungsverträge vom Geltungsbereich des Art. 5a Rom-I-Vorschlag auszunehmen und dem allgemeinen Anknüpfungsregime der Art. 3, 4 Rom-I-Vorschlag zu unterstellen.

\subsubsection{Integration von Großrisikoversicherungen}

Auch die Regelung der Großrisikoversicherungen führt nur zu einer formalen Integration dieses Regelungsgegenstandes in Art. 5a Rom-I-Vorschlag. Inhaltlich aber werden Großrisikoversicherungen nicht nur vom zwingenden Charakter des Art. 5a befreit, sondern auch einer von anderen Versicherungsverträgen abweichenden objektiven Anknüpfung an den gewöhnlichen Aufenthalt des Versicherers unterworfen. Besser wäre es, für Großrisiken keine abweichende objektive Anknüpfung zu wählen, sondern sie nur von der zwingenden Anknüpfung des Art. 5a zu befreien. Damit wäre auch der Gleichlauf zur EuGVVO und zum nationalen (z. B. deutschen) Versicherungsvertragsrecht hergestellt. ${ }^{80}$

Bei allem definiert Art. 5a Abs. 1a das Großrisiko durch Verweis auf das Richtlinienrecht. Besser wäre, wie dargelegt ${ }^{81}$, ein Verweis auf Art. 13 Abs. 5 i.V.m. Art. 14 EuGVVO und eine Neudefinition des Großrisikos ebendort.

\subsubsection{Rechtswahl bei Massenrisikoversicherung}

Bei der Versicherung von Massenrisiken räumt das Papier der Präsidentschaft in seiner ursprünglichen Fassung den Parteien eine beschränkte Rechtswahlmöglichkeit ein. ${ }^{82}$ Im Wesentlichen geht es um zwei Situationen beschränkter Parteiautonomie: Zum einen können die Parteien in den Fäl-

79 Zur Frage und im Sinne des Papiers der Ratspräsidentschaft Mankowski, Internationales Rückversicherungsvertragsrecht, VersR 2002, 1177.

80 Hierzu schon oben 2.2.4.

81 Hierzu oben 2.2.4.

82 Siehe den vorgeschlagenen Art. 5a Abs. 3 im Papier der Ratspräsidentschaft. 
len sogenannter Divergenz, also des Auseinanderfallens von gewöhnlichem Aufenthalt des Versicherungsnehmers und Belegenheit des Risikos, das Recht des gewöhnlichen Aufenthalts des Versicherungsnehmers anstelle des nach gesetzlicher Anknüpfung zur Anwendung berufenen Rechts am Ort der Risikobelegenheit wählen. ${ }^{83}$ Zum anderen können die Parteien eines Lebensversicherungsvertrags bei Auseinanderfallen von gewöhnlichem Aufenthaltsort und Staatsbürgerschaft des Versicherungsnehmers das Recht der Staatsangehörigkeit des Versicherungsnehmers zum Vertragsstatut küren. ${ }^{84}$

Diese beschränkten Rechtswahlmöglichkeiten reichen zwar weiter als die Änderungsanträge der Parlamentarier, doch greifen sie immer noch deutlich zu kurz: Insbesondere erlaubt der Vorschlag keine freie Rechtswahl bei aktiv grenzüberschreitender Nachfrage seitens des Versicherungsnehmers. Insofern ändern auch die Nachbesserungen im Papier vom 4. 5. 2007 nur wenig an diesem Befund. Trotz deutlicher Erweiterung der Rechtswahlmöglichkeiten fehlt eine Rechtswahl des aktiven Versicherungsnehmers. An einer Stelle $^{85}$ - es geht um die Anknüpfung von Pflichtversicherungen - verweist der neue Ratsvorschlag für den Umfang der Rechtswahlfreiheit sogar auf das nationale Kollisionsrecht, führt also bewusst keine Kollisionsrechtsvereinheitlichung herbei und greift damit jenen Ansatz wieder auf, der bereits nach dem bestehenden europäischen Kollisionsrecht Anlass zur Kritik gibt.

Weiterhin zwingt die Verwendung des Begriffs der Risikobelegenheit dazu, in Art. 5a Abs. 5 eine Definition einzufügen, die notwendigerweise nicht einfach ausfällt. Dabei zeigt ein Blick in die gewählte, dem Richtlinienrecht folgende Definition, dass der gewöhnliche Aufenthalt des Versicherungsnehmers und die Risikobelegenheit im Regelfall eins sind. In aller Regel ist damit eine Rechtswahl nach Art. 5a Abs. 3 S. 2 (Divergenzfall) gerade nicht eröffnet. Man fragt sich daher, ob das Anliegen dieser Regelung nicht einfacher und besser erreichbar wäre.

\subsubsection{Streichung von Art. 3 Abs. 2 und Art. 22 lit b) Rom-I-Vorschlag}

Das Papier der Ratspräsidentschaft streicht Art. 3 Abs. 2 Rom-I-Vorschlag (Wahl „nichtstaatlichen Rechts“) sowie Art. 22 lit b) Rom-I-Vorschlag (Vorbehalt eines zukünftigen optionalen Instruments). ${ }^{86}$ Diese Änderungen sind zutiefst bedauerlich. Für die Schaffung eines optionalen Instruments sprechen nämlich gerade im Versicherungsvertragsrecht alle

83 Siehe den vorgeschlagenen Art. 5a Abs. 3 S. 2 im Papier der Ratspräsidentschaft.

84 Siehe den vorgeschlagenen Art. 5a Abs. 3 S. 3 im Papier der Ratspräsidentschaft.

85 Siehe Art. 5a Abs. 2 S. 2 im Papier der Ratspräsidentschaft i. d. F. 4. 5. 2007.

86 Siehe die vorgeschlagenen Art. 3 und 22 im Papier der Ratspräsidentschaft. 
Gründe: Erstens die Bedürfnisse des Binnenmarkts, zweitens der Umstand, dass nur der europäische Gesetzgeber ein solches schaffen kann, und drittens die Tatsache, dass nationale Interessen nicht entgegenstehen, weil in die nationalen Versicherungsvertragsrechte ohnehin nicht eingegriffen wird. ${ }^{87}$ Es leuchtet nicht ein, warum sich der Rat dagegen sträubt, Vertragsparteien im Binnenmarkt eine für sie wesentliche Option einzuräumen. Man darf hoffen, dass sich am Ende die besseren Argumente auch im Rat durchsetzen.

\subsection{Gelungene Teilregelung des internationalen Versicherungsvertragsrechts durch die Rom-II-Verordnung Direktklage und Regressrecht}

Während sich nach alledem die geplante Rom-I-Verordnung noch mitten in der Diskussion befindet, wurde die Rom-II-Verordnung bereits erlassen. ${ }^{88}$ Sie enthält Regelungen, die auch für das internationale Versicherungsvertragsrecht von Bedeutung sind. Das betrifft zunächst Art. 18 RomII-Verordnung, der die Direktklage gegen den Versicherer des Haftenden regelt. Demnach kommt dem Geschädigten ein Direktklagerecht alternativ immer dann zu, wenn entweder das Versicherungsvertragsstatut oder aber das Deliktsstatut eine solche Direktklage vorsehen. Weiterhin ist Art. 19 Rom-II-Verordnung zu nennen, der das gesetzliche Regressrecht betrifft. Demnach entscheidet das Versicherungsvertragsstatut, ob und in welchem Umfang die Forderung des Versicherungsnehmers gegen den haftenden Dritten auf den Versicherer übergeht.

\section{Reformvorschlag des Max-Planck-Instituts für ausländisches und internationales Privatrecht vom 16. Juni $\mathbf{2 0 0 6}^{89}$, Hamburg}

\subsection{Vorbemerkung}

Die Entwicklungen zum Rom-I-Vorschlag wurden von Stellungnahmen aus der Wissenschaft begleitet und teilweise auch befruchtet. Unter allen ist die umfassende Abhandlung des Max-Planck-Instituts für ausländisches

87 Nachweise bei Heiss, Stand und Perspektiven der Harmonisierung des Versicherungsvertragsrechts in der EG (2005) $16 \mathrm{ff}$. und $36 \mathrm{ff}$.

88 VO (EG) Nr. 864 / 2007 des EP und des Rates vom 1. Juli 2007 über das auf außervertragliche Schuldverhältnisse anzuwendende Recht, ABl. 2007 Nr. L 199/40; hierzu jüngst Heiss / Loacker, Die Vergemeinschaftung des Kollisionsrechts der außervertraglichen Schuldverhältnisse durch Rom II, JBl. 2007, 613.

89 Max Planck Institute for Comparative and International Private Law, Comments on the European Commission's Proposal for a Regulation of the European Parliament and the Council on the law applicable to contractual obligations (Rome I), RabelsZ 71 (2007) 225; einsehbar auch unter http://www.mpipriv-hh.mpg.de; im Folgenden zitiert als: MPI-Vorschlag, RabelsZ 71 (2007) 225. 
und internationales Privatrecht in Hamburg vom 16. Juni 2006 hervorzuheben. Das Max-Planck-Institut geht hierin auch auf die Anknüpfung von Versicherungsverträgen ein.

\subsection{Integration des Binnenmarktkollisionsrechts für Versicherungsverträge}

Auch das Max-Planck-Institut spricht sich deutlich für eine Integration des Binnenmarktkollisionsrechts für Versicherungsverträge in die Rom-IVerordnung aus. ${ }^{90}$ Vieles spricht dafür, dass die jüngeren Änderungsanträge der Parlamentarier und auch das Papier der Ratspräsidentschaft insofern dem Vorschlag des Max-Planck-Instituts gefolgt sind.

\subsection{Versicherungsnehmerschutz, Rück- und Großrisikoversicherungen}

Der vom Max-Planck-Institut vorgeschlagene Art. 5a Rom-I-Verordnung verfolgt den Ansatz, auch gewerbliche Versicherungsnehmer zu schützen. Das Max-Planck-Institut nimmt diesen Schutz nur für Rück- und Großrisikoversicherungen zurück. Der vorgeschlagene Art. 5a soll nämlich weder auf Rück- noch auf Großrisikoversicherungen anwendbar sein. ${ }^{91}$ Dem ist für Rückversicherungen zuzustimmen. Für Großrisikoversicherungen sind hingegen die bereits dargelegten Bedenken zu wiederholen: ${ }^{92}$ Die Ausnahme vom Anwendungsbereich des Art. 5a widerspricht dem Ansatz der EuGVVO, den PEICL und auch dem deutschen Versicherungsvertragsrecht. Es ist auch kein Grund erkennbar, warum die objektive Anknüpfung von Großrisikoversicherungen anders ausfallen sollte als im Massengeschäft. Jedenfalls sollte die von Art. 5a zwingend gestellte Anknüpfung von Pflichtversicherungsverträgen auch bei Großrisikoversicherungen gelten. Besser wäre somit eine Geltungserstreckung von Art. 5a auch auf Großrisikoversicherungen, allerdings unter Rücknahme des zwingenden Charakters der Vorschrift.

Zum Zwecke der Definition des Großrisikos verweist die Stellungnahme des Max-Planck-Instituts auf das Richtlinienrecht. ${ }^{93}$ Im Sinne eines perfekten Gleichlaufs der Rechtswahlfreiheit mit der Freiheit der Gerichtsstandswahl wäre es demgegenüber vorzugswürdig, auf Art. 13 Abs. 5 i.V.m. Art. 14 EuGVVO zu verweisen und gleichzeitig die dort gegebene Definition neu zu fassen. ${ }^{94}$

90 Siehe Art. 5a MPI-Vorschlag, RabelsZ 71 (2007) 225 (277 f.).

91 Siehe Art. 5a Abs. 4 MPI-Vorschlag, RabelsZ 71 (2007) 225 (278).

92 Siehe oben 2.2.4.

93 Siehe Art. 5a Abs. 4 MPI-Vorschlag, RabelsZ 71 (2007) 225 (278).

94 Siehe oben 2.2.4. 


\subsection{Objektive Anknüpfung}

Für die objektive Anknüpfung stellt das Max-Planck-Institut auf den gewöhnlichen Aufenthalt des Versicherungsnehmers im Zeitpunkt des Vertragsschlusses ab. Dieses Anknüpfungsmuster ist doppelt zu begrüßen: Zum einen erscheint das Anknüpfungsmoment des gewöhnlichen Aufenthalts als sachgerecht und praktisch einfach handhabbar. Zum anderen macht der Hinweis auf den Vertragsschlusszeitpunkt einen späteren Statutenwechsel unerheblich.

Die Stellungnahme des Max-Planck-Instituts stellt indessen neben dem gewöhnlichen Aufenthalt auch auf den Sitz der Hauptverwaltung des Versicherungsnehmers ab. ${ }^{95}$ Der Bedarf nach einer solchen Regelung erscheint allerdings zumindest fraglich. Art. 18 Rom-I-Vorschlag und auch Art. 18 des Vorschlags des Max-Planck-Instituts stellen nämlich ohnehin den Ort der Hauptniederlassung bzw. der Zweigniederlassung etc. dem gewöhnlichen Aufenthalt gleich. Dies sollte auch für die Zwecke des Art. 5a ausreichen.

\subsection{Rechtswahl}

Im Vergleich zu den Änderungsanträgen der Parlamentarier wie auch zum Papier der Ratspräsidentschaft schlägt das Max-Planck-Institut einen deutlich weiteren Rahmen an Rechtswahlmöglichkeiten der Vertragsparteien vor. ${ }^{96}$ Demnach sollen die Parteien wählen können:

a) in den Fällen der Divergenz, also des Auseinanderfallens von gewöhnlichem Aufenthalt des Versicherungsnehmers und Belegenheit des Risikos, das Recht der Risikobelegenheit;

b) für Versicherungsverträge über Versicherungsfälle, die auf einen bestimmten Staat beschränkt sind, das Recht dieses Staates;

c) im Bereich der Lebensversicherung das Recht der Staatsangehörigkeit des Versicherungsnehmers;

d) bei kurzfristigen Reise- bzw. Urlaubsversicherungen das Recht des Staates, in dem der Versicherungsnehmer die Police genommen hat.

Diese Ausweitungen der Rechtswahlmöglichkeiten sind gewiss zu begrüßen. Man darf nämlich nicht übersehen, dass die Wahl zugunsten bestimmter Rechte immer auch die Öffnung dieser Märkte für die Nachfrager (Versicherungsnehmer) bedeutet. Dies ist generell zwar wegen des erforderlichen Versicherungsnehmerschutzes nicht duldbar, sollte aber in den echten Bedarfsfällen nicht verweigert werden. Genau darum geht es dem

95 Siehe Art. 5a Abs. 1 MPI-Vorschlag, RabelsZ 71 (2007) 225 (277).

96 Siehe Art. 5a Abs. 2 MPI-Vorschlag, RabelsZ 71 (2007) 225 (277). 
Max-Planck-Institut ganz offenbar, woraus sich die Liste der vier Rechtswahlmöglichkeiten ergibt.

Dennoch stellt der Katalog der Rechtswahlmöglichkeiten keine hinreichende Lösung des Rechtswahlproblems dar. Nach dem Vorschlag des MaxPlanck-Instituts ist es dem Versicherungsnehmer nämlich in allen nicht gelisteten Fällen auch dann verwehrt, eine freie Rechtswahl zu treffen, wenn er aktiv grenzüberschreitend Versicherungsschutz nachfragt. In diesen Fällen stellt aber sogar Art. 5 Rom-I-Vorschlag dem privaten Endverbraucher die freie Rechtswahl zur Verfügung. ${ }^{97}$ Auch wurde bereits dargetan, dass eine solche Rechtswahl im Interesse von Versicherungsnehmern, die am heimischen Markt keinen geeigneten Versicherungsschutz finden, dringend geboten erscheint. ${ }^{98}$

\subsection{Definition der Risikobelegenheit}

Die Regelungen zur Rechtswahl verwenden auch den Begriff der Belegenheit des Risikos. ${ }^{99}$ Zum Zwecke seiner Definition verweist der Vorschlag des Max-Planck-Instituts auf das Recht des Forumstaates. ${ }^{100}$ Damit garantiert der Vorschlag keine einheitliche europäische Begriffsbildung. Eine solche Garantie liegt nur solange vor, wie die Definition der Risikobelegenheit in den Versicherungsrichtlinien und damit in den nationalen Umsetzungsgesetzen enthalten ist. Nach Integration des internationalen Versicherungsvertragsrechts in eine Rom-I-Verordnung wird es für Zwecke des Kollisionsrechts einer Definition der Belegenheit im Richtlinienrecht aber nicht mehr bedürfen. Sie wird im Richtlinienrecht nur noch für andere Zwecke, also für diverse aufsichts- ${ }^{101}$ und steuerrechtliche ${ }^{102}$ Regelungen, benötigt, wobei der Begriff im Steuerrecht von der allgemeinen Definition abweicht. ${ }^{103}$ Für den deutschen Gesetzgeber bieten sich daher nur zwei Mög-

97 So auch Staudinger, Internationales Versicherungsvertragsrecht, in: Ferrari/ Leible (Hrsg.), Ein neues Internationales Vertragsrecht für Europa (2007) 225 (241).

98 Hierzu oben 2.2.5.

99 Kritisch insofern Staudinger, Internationales Versicherungsvertragsrecht, in: Ferrari / Leible (Hrsg.), Ein neues Internationales Vertragsrecht für Europa (2007) 225 (240).

100 Siehe Art. 5a Abs. 2 (a) MPI-Vorschlag, RabelsZ 71 (2007) 225 (277).

101 Die aufsichtsrechtlichen Regelungen verwenden z. B. den Begriff des „Mitgliedstaats der Dienstleistung“ (z. B. Art. 38, 39 Dritte RL Nicht-Lebensversicherung [ABl. 1992 Nr. L 228/1]), der in Art. 1 lit e) der RL als der Mitgliedstaat der Risikobelegenheit im Sinne des Art. 2 lit d) der Zweiten RL Nicht-Lebensversicherung (ABl. 1988 Nr. L 172 / 1) definiert wird.

102 Siehe Art. 46 Abs. 2 Dritte RL Nicht-Lebensversicherung (ABl. 1992 Nr. L $228 / 1)$.

103 Siehe Art. 46 Abs. 2 Unterabs. 2 Dritte RL Nicht-Lebensversicherung (ABl. 1992 Nr. L 228 / 1), der für steuerrechtliche Zwecke eine von Art. 2 Buchstabe d) abweichende Teildefinition gibt. 
lichkeiten: Er könnte zum einen die kollisionsrechtliche Definition in Art. 7 EGVVG bei gleichzeitiger Streichung der Kollisionsnormen der Art. 8-15 EGVVG (diese würden ja durch Art. 5a Rom-I-Verordnung ersetzt) beibehalten. Dann aber fragt man sich, wieso eine derart isolierte Definition im nationalen Recht beibehalten und nicht mit den Kollisionsnormen zusammen in die Rom-I-Verordnung übernommen werden soll. Der deutsche Gesetzgeber könnte zum anderen Art. 7 EGVVG streichen. Dann müsste der deutsche Richter bei Anwendung des vorgeschlagenen Art. 5 a Abs. 2 lit. a) Rom-I-Verordnung die Definition der Risikobelegenheit in $\S 13$ a Abs. 2 S. 2 VAG (Versicherungsgeschäfte über Niederlassungen oder im Dienstleistungsverkehr) finden. Dies würde die Rechtsanwendung verkomplizieren. Es wäre daher wohl vorzugswürdig, die Risikobelegenheit, wenn denn der Begriff im Anknüpfungsregime eine Rolle spielen soll ${ }^{104}$, in der Rom-I-Verordnung eigens zu definieren.

\section{Eigener Vorschlag \\ 4.1 Integration des Binnenmarktkollisionsrechts für Versicherungsverträge}

Auch der hier vorgestellte Entwurf folgt dem Prinzip, das internationale Versicherungsvertragsrecht abschließend in der vorgeschlagenen Rom-IVerordnung zu regeln. Von den dargestellten Entwürfen abweichend, wird hier allerdings ein Art. 6 Rom-I-Verordnung vorgeschlagen, was zur Folge hätte, dass die nachfolgenden Artikel des Rom-I-Vorschlags neu zu nummerieren wären.

\subsection{Vorschlag eines Art. 6 Rom-I-Verordnung}

\section{Art. 6. Versicherungsverträge.}

(1) Versicherungsverträge, mit Ausnahme von Rückversicherungsverträgen, unterliegen dem Recht des Staates, in dem der Versicherungsnehmer im Zeitpunkt des Vertragsabschlusses seinen gewöhnlichen Aufenthalt hat.

(2) Abweichend von Absatz 1 können die Parteien das anzuwendende Recht [bzw. die anzuwendenden Grundsätze und Regeln im Sinne des Artikels 3 Absatz 2] frei wählen, wenn:

a) der Versicherungsvertrag besondere oder große Risiken im Sinne von Artikel 13 Absatz 5 in Verbindung mit Artikel 14 der Verordnung (EG) Nr. 44 / 2001 des Rates über die gerichtliche Zuständigkeit und die Anerkennung und Vollstreckung von Entscheidungen in Zivil- und Handelssachen vom 22. Dezember 2000 (ABl. 2001 Nr. L 12 / 1) in der jeweils geltenden Fassung deckt, oder

104 Siehe demgegenüber den eigenen Vorschlag unten IV.2., insbesondere den vorgeschlagenen Art. 6 Abs. 1. 
b) der Versicherungsvertrag mit einem Versicherer geschlossen wird, der im Staat des gewöhnlichen Aufenthalts des Versicherungsnehmers das Versicherungsgeschäft weder selbst noch durch Mittelspersonen betreibt und auch nicht auf irgendeinem Wege auf diesen Staat ausgerichtet hat oder der Vertragsschluss in keinem Zusammenhang mit einer solchen Geschäftstätigkeit des Versicherers steht.

(3) a) Gruppenversicherungsverträge unterliegen dem Recht des Staates, in dem die Gruppenmitglieder ihren gewöhnlichen Aufenthalt haben. Werden Gruppenmitglieder mit gewöhnlichem Aufenthalt in verschiedenen Staaten versichert, so ist der Vertrag so zu behandeln, als bestünde er aus mehreren Verträgen, von denen sich jeder auf jeweils einen Mitgliedstaat bezieht.

b) Eine zwischen Versicherer und Gruppenorganisator getroffene Rechtswahl nach Absatz 2 ist mit Wirkung für die Gruppenmitglieder nur wirksam, soweit

- der Gruppenversicherungsvertrag besondere Risiken im Sinne des Absatzes 2 lit a) versichert,

- die Gruppenmitglieder die subjektiven Kriterien für Großrisiken im Sinne des Absatzes 2 lit a) erfüllen oder

- zusätzlich zu den Voraussetzungen des Absatzes 2 lit b) das Gruppenmitglied einer Gruppe beitritt, deren Organisator seine ideelle oder gewerbliche Tätigkeit im Staat des gewöhnlichen Aufenthalts des Gruppenmitglieds weder ausübt noch auf irgendeinem Wege auf diesen Staat ausgerichtet hat oder der Beitritt in keinem Zusammenhang mit einer solchen Tätigkeit des Organisators steht.

c) Wird die Gruppenversicherung von einem Arbeitgeber für Risiken seiner Arbeitnehmer genommen, so können die Parteien das Recht des Staates wählen, in dem der Arbeitgeber seine Hauptniederlassung oder jene Betriebsstätte hat, an der oder von der aus der Arbeitnehmer seine Arbeit gewöhnlich verrichtet.

(4) Pflichtversicherungsverträge unterliegen dem Recht des Staates, der die Versicherungspflicht vorschreibt.

(5) Krankenversicherungsverträge, die eine im gesetzlichen Sozialversicherungssystem eines Staates vorgesehene Krankenversicherung ganz oder teilweise ersetzen, unterliegen dem Recht dieses Staates.

\subsection{Versicherungsnehmerschutz}

Der Vorschlag schützt nicht nur private Endverbraucher, sondern auch gewerblich tätige Versicherungsnehmer. Er macht dabei nur zwei Ausnahmen: Die Rückversicherung wird gemäß Art. 6 Abs. 1 vom Anwendungsbereich der Regelung nicht erfasst. Großrisikoversicherungen unterliegen zwar der Regelung des Art. 6, bei ihnen besteht jedoch freie Rechtswahl (Art. 6 Abs. 2 lit. a). Art. 6 Abs. 2 lit. a verweist auf die Definition der besonderen und großen Risiken nach Art. 13 Abs. 5 i.V.m. Art. 14 der EuGVVO. Dadurch wird der persönliche Anwendungsbereich des Versicherungsnehmerschutzes in völligen Einklang mit dem internationalen Zuständigkeitsrecht gebracht. Die Definition des Großrisikos in der EuGVVO bedarf freilich ihrerseits einer Reform, die Hand in Hand mit dem Erlass der RomI-Verordnung erfolgen sollte. 


\subsection{Rechtswahl}

\subsubsection{Freie Rechtswahl}

Nach dem hier unterbreiteten Vorschlag kommt den Vertragsparteien immer dann freie Rechtswahl zu, wenn für einen besonderen Versicherungsnehmerschutz kein Anlass besteht. Dies gilt in den Fällen der Rückversicherungsverträge, die insoweit der freien Rechtswahl nach Art. 3 Rom-I-Vorschlag unterstehen, aber auch für Großrisikoversicherungen, für die Art. 6 Abs. 2 lit. a des Vorschlags die freie Rechtswahl einräumt. Die Fälle der freien Rechtswahl werden darüber hinaus in Anlehnung an Art. 5 (Verbraucherverträge) in Art. 6 Abs. 2 lit. b auf den Fall ausgedehnt, in dem ein Versicherungsnehmer aktiv grenzüberschreitend ausländischen Versicherungsschutz nachfragt. ${ }^{105}$ Dies erfolgt nicht nur aus Gründen einer systematischen Übereinstimmung mit Art. 5, sondern weil ein unmittelbarer Bedarf nach einer solchen Rechtswahlmöglichkeit in der Kritik der zuvor dargestellten Vorschläge bereits deutlich wurde. ${ }^{106}$ Art. 6 übernimmt die Regelung des Art. 5 Rom-I-Vorschlag nicht wörtlich, sondern strebt durch die Einfügung der Wortfolge „...oder der Vertragsschluss in keinem Zusammenhang mit einer solchen Geschäftstätigkeit des Versicherers steht" eine Verdeutlichung des Regelungsgehalts an. Damit wird dem Grundsatz des Art. 28 der Dritten Richtlinie Nicht-Lebensversicherung ${ }^{107}$, wonach der Mitgliedstaat der Risikobelegenheit den Versicherungsnehmer nicht am Vertragsschluss mit im EWR-Ausland niedergelassenen Versicherern hindern darf, auch kollisionsrechtlich entsprochen.

\subsubsection{Entfall der Liste an beschränkten Rechtswahlmöglichkeiten}

Die freie Rechtswahl des aktiv grenzüberschreitend nachfragenden Versicherungsnehmers erübrigt $\mathrm{m}$. E. eine kasuistisch anmutende Auflistung von einzelnen Rechtswahlmöglichkeiten. ${ }^{108}$

Ganz augenscheinlich bedarf es in den Fällen der Reise- bzw. Urlaubsversicherungen keiner weiteren Rechtswahlmöglichkeit. Hier ist an eine Versicherungsnahme auf der Reise (etwa anlässlich eines Zwischenstopps auf einem Flughafen) gedacht. Befindet sich der Versicherungsnehmer bei Abschluss im Ausland, so würde ihm nach dem hier vertretenen Konzept ohnehin die freie Rechtswahl des aktiv grenzüberschreitenden Nachfragers zu-

105 So auch Staudinger, Internationales Versicherungsvertragsrecht, in: Ferrari/ Leible (Hrsg.), Ein neues Internationales Vertragsrecht für Europa (2007) 225 (241).

106 Siehe insbesondere oben 2.2.5, 2.3.4, 3.5.

107 ABl. $1992228 / 1$.

108 Kritisch zu einer Auflistung einzelner Rechtswahlmöglichkeiten auch Staudinger, Internationales Versicherungsvertragsrecht, in: Ferrari / Leible (Hrsg.), Ein neues Internationales Vertragsrecht für Europa (2007) 225 (240). 
kommen. Schließt der Versicherungsnehmer die Versicherung demgegenüber zu Hause ab, so hat er auch nach dem MPI-Vorschlag keine Rechtswahlmöglichkeit, weil Rechtswahl und objektive Anknüpfung dann zu ein und demselben Recht führen.

Ähnliches gilt für die Rechtswahl in den Divergenzfällen. Versichert etwa ein Versicherungsnehmer mit gewöhnlichem Aufenthalt in Deutschland ein in Spanien gelegenes Haus, so bieten sich ihm vornehmlich zwei Möglichkeiten: Wendet er sich an einen deutschen Versicherer, so wird ihm die Rechtswahlmöglichkeit zugunsten der Belegenheit des Hauses, also zugunsten spanischen Rechts, kaum helfen. Wenn der deutsche Versicherer überhaupt geneigt sein wird, das in Spanien belegene Risiko zu versichern, so wird er dem Vertrag deutsches Recht zugrunde legen. Genau gegenteilig stellt sich die Sachlage dar, wenn der Versicherungsnehmer von seiner zweiten Möglichkeit Gebrauch macht. Er könnte sich nämlich an seinen spanischen Versicherer wenden. Dieser wird das Haus gerne in Deckung nehmen, allerdings nur unter Zugrundelegung spanischen Rechts. In diesen Fällen ist der Versicherungsnehmer aber ohnehin ein aktiv grenzüberschreitender Nachfrager. Ihm kommt also nach dem hier vertretenen Konzept die Rechtswahl zu. Die Rechtswahloption in den Divergenzfällen kann beim hier vertretenen Konzept also entfallen. Mit dem Entfall erspart man sich zugleich die Verwendung und damit auch die Definition des Begriffs der Risikobelegenheit. ${ }^{109}$

Die gleichen Erwägungen treffen den Fall, in dem eine Versicherungspolice nur Versicherungsfälle deckt, die in einem bestimmten Staat eintreten. Deckt etwa ein Versicherungsnehmer mit gewöhnlichem Aufenthalt in Deutschland sein US-amerikanisches Produkthaftungsrisiko bei einem deutschen Versicherer ab, so wird er dies nur unter Zugrundelegung deutschen Versicherungsrechts tun. Will sich der Versicherungsnehmer des USamerikanischen Versicherungsmarkts bedienen, so kann er dies, weil er als aktiv grenzüberschreitender Nachfrager die Rechtswahlfreiheit genießt. Offen bleibt lediglich der Fall, in dem US-amerikanische Versicherer ihre nach US-amerikanischem Recht entwickelten Produkthaftpflicht-Policen am deutschen Markt vertreiben wollen. Genau in diesem Falle aber greift der Schutzgedanke des vorgeschlagenen Art. 6, sodass die Rechtswahlmöglichkeit gerade nicht eröffnet sein sollte.

$\mathrm{Zu}$ erörtern bleibt noch die Wahlmöglichkeit zugunsten des Rechts der Staatsangehörigkeit des Versicherungsnehmers in der Lebensversicherung. Nach dem hier vertretenen Vorschlag kann etwa ein Franzose mit gewöhn-

109 Das setzt freilich weiter voraus, dass auch die objektive Anknüpfung nicht auf das Moment der Belegenheit des Risikos abstellt; hierzu Art. 6 Abs. 1 des Vorschlags, der auf den gewöhnlichen Aufenthalt des Versicherungsnehmers und nicht auf die Risikobelegenheit abstellt. 
lichem Aufenthalt in Deutschland seine Lebensversicherung im Wege aktiv grenzüberschreitender Nachfrage bei einem französischen Versicherer und nach französischem Recht abschließen. Bei einem deutschen Versicherer kommt ihm diese Rechtswahlmöglichkeit nicht zu, doch würde sich der deutsche Versicherer auf eine solche ohnehin nicht einlassen. Offen bleiben hier lediglich die Fälle, in denen französische Versicherer ihre Lebensversicherungspolicen an französische Staatsangehörige, die im Ausland leben, vertreiben wollen. In diesen Fällen handelt der Versicherungsnehmer nicht aktiv grenzüberschreitend, sodass ihm keine Rechtswahlmöglichkeit zukommt. Das entspricht ja gerade dem Schutzkonzept des hier vorgeschlagenen Art. 6 Rom-I-Verordnung. Es fragt sich allerdings, ob das Fehlen dieser Rechtswahlmöglichkeit die Nachfragefreiheit des französischen Versicherungsnehmers ungebührend beschneidet. Das wäre dann zu befürchten, wenn der Schutz, wie er durch die zwingende Anwendung des am gewöhnlichen Aufenthaltsort des Versicherungsnehmers geltenden Rechts gewährt wird, in diesen Fällen typischerweise nicht notwendig ist. Dies würde voraussetzen, dass ein in Deutschland lebender französischer Versicherungsnehmer regelmäßig zur Sprache und zum Recht seines Herkunftslandes mindestens die gleich enge Beziehung aufweist wie zum deutschen Recht. Dabei ist auch zu berücksichtigen, ob ihm der Zugang zum Recht (z. B. die Auswahl eines geeigneten Rechtsanwalts) in Frankreich genauso leicht fällt wie in Deutschland. ${ }^{110}$ Man mag diese Frage mit Blick auf den durch die Freizügigkeit ermöglichten „euromobilen“ Bürger bejahen. ${ }^{111}$ Insofern spricht die Freizügigkeit ${ }^{112}$ im Binnenmarkt für eine Option zugunsten des Rechts der Staatsangehörigkeit des Versicherungsnehmers. Sie spricht aber, genau gesehen, auch und m. E. noch stärker dagegen: Die Staatsangehörigkeit eines EU/EWR-Ausländers lässt nämlich gerade wegen der in der EU/im EWR gewährten Freizügigkeit keinen annähernd verlässlichen Rückschluss auf die Verbundenheit des Ausländers mit seinem Herkunftsstaat zu. Die Freizügigkeit garantiert dem EU/EWR-Ausländer insbesondere eine im Vergleich zu den Angehörigen des Aufenthaltsstaates nicht diskriminierende Behandlung. ${ }^{113}$ Es fehlt daher, selbst bei Verbleib im Aufenthaltsstaat, an einem besonderen Anreiz, die Staatsangehörigkeit des Herkunftsstaates zugunsten jener des Aufenthaltsstaates aufzugeben. In den Mitgliedstaaten leben somit viele EU/EWR-Ausländer, die den Staat,

110 Ähnlich weist auch Gruber, Internationales Versicherungsvertragsrecht (1999) 5 auf dieses Problem hin.

111 Zum Begriff Basedow, Das österreichische Bundesgesetz über internationales Versicherungsvertragsrecht, in: Reichert-Facilides (Hrsg.), Aspekte des internationalen Versicherungsvertragsrechts im Europäischen Wirtschaftsraum (1994) 89 (99 f.).

112 Art. 18 EGV (Allgemeine Freizügigkeit) und Art. 39 EGV (Freizügigkeit der Arbeitnehmer).

113 Vgl. insb. Art. 39 Abs. 2 EGV; im Übrigen gilt das allgemeine Diskriminierungsverbot des Art. 12 EGV. 
welchem sie angehören, vor langer Zeit verlassen haben, ja zum Teil nicht einmal dort geboren wurden. Insbesondere Doppelstaatsbürger zählen hierher. Sie bedürfen des kollisionsrechtlichen Versicherungsnehmerschutzes wie Inländer. Dieser aber würde durch eine generelle Wahlmöglichkeit zugunsten des Rechts der Staatsangehörigkeit unterlaufen. Diejenigen EU / EWR-Ausländer, die einen engen Kontakt zur Heimat pflegen, werden von der Rücknahme dieser Rechtswahloption nicht unzumutbar belastet. Es steht ihnen nämlich nach dem hier vorgeschlagenen Art. 6 Abs. 2 lit. b) Rom-I-Verordnung jederzeit frei, in ihrem Heimatstaat Versicherungsschutz nach dem dort geltenden Recht aktiv grenzüberschreitend nachzufragen. Gerade jenen EU/EWR-Ausländern, die engen Kontakt in ihre Heimat pflegen, wird dies auch tatsächlich nicht schwer fallen. Auch in diesen Konstellationen genügt somit die freie Rechtswahl des aktiv grenzüberschreitend nachfragenden Versicherungsnehmers.

\subsubsection{Wahl von anerkannten Grundsätzen und Regeln des Vertragsrechts i.S.d. Art. 3 Abs. 2 Rom-I-Vorschlag}

Die hier vertretene Regelung der Rechtswahl wurde noch mit Blick auf den von der Kommission vorgeschlagenen Art. 3 Abs. 2 Rom-I-Verordnung ausformuliert. Die Regelung erwähnt daher neben der Möglichkeit, ein (staatliches) Recht zu wählen, auch die Option, den Versicherungsvertrag Grundsätzen und Regeln im Sinne des Art. 3 Abs. 2 zu unterstellen. ${ }^{114}$ Dieser Hinweis müsste entfallen, wenn eine solche Wahlmöglichkeit zugunsten nichtstaatlichen Rechts in der Rom-I-Verordnung fehlen sollte. Dass dies bedauerlich und falsch wäre, wurde bereits oben dargelegt. ${ }^{115}$

\subsection{Gesetzliche Anknüpfung}

Mangels Rechtswahl knüpft Art. 6 Abs. 1 Versicherungsverträge (mit Ausnahme von Rückversicherungsverträgen) an den gewöhnlichen Aufenthalt des Versicherungsnehmers im Zeitpunkt des Vertragsschlusses an. Auch hier entfällt jegliche Bezugnahme auf den Terminus der Risikobelegenheit, sodass seine Definition in dem Regelungsvorschlag insgesamt fehlt.

\subsection{Pflichtversicherung}

Der hier vorgestellte Vorschlag unterwirft Pflichtversicherungsverträge dem Recht des Staates, der die Versicherungspflicht vorschreibt (Art. 6 Abs. 4). Er folgt also den zuvor vorgestellten Entwürfen.

114 Siehe Art. 6 Abs. 2 des Vorschlags.

115 Hierzu oben 2.3.lit 5. 


\subsection{Substitutive Krankenversicherung}

Der hier vorgestellte Vorschlag (Art. 6 Abs. 5) ergänzt die zuvor diskutierten Entwürfe mit Blick auf die substitutive Krankenversicherung. ${ }^{116}$ Aus ähnlichen Erwägungen wie bei den Pflichtversicherungsverträgen sollen diese Verträge dem Recht des Staates unterworfen werden, dessen Sozialversicherungssystem durch die private Krankenversicherung ganz oder teilweise ersetzt wird. Eine derartige Regelung rechtfertigt sich $\mathrm{m}$. E. durch Art. 54 der Dritten Richtlinie Nicht-Lebensversicherung ${ }^{117}$. Diese Vorschrift erlaubt es den Mitgliedstaaten, auf substitutive Krankenversicherungen die ,spezifischen Rechtsvorschriften zum Schutz des Allgemeininteresses in Bezug auf diesen Versicherungszweig“" anzuwenden. Dies erfordert zwar nicht notwendig eine Unterwerfung des gesamten Krankenversicherungsvertrags dem Recht des Staates, dessen Sozialversicherungssystem substituiert wird, eine solche Regelung empfiehlt sich aber im Sinne der Einfachheit. Diesen Gedanken nimmt Art. 6 Abs. 5 auf.

\subsection{Gruppenversicherung}

\subsubsection{Allgemeines}

Alle zuvor diskutierten Entwürfe leiden m. E. unter dem Mangel, dass sie Gruppenversicherungsverträge nicht spezifisch regeln. Das gilt namentlich für jene Typen von Gruppenversicherungen, bei denen das einzelne Gruppenmitglied nicht Versicherungsnehmer, sondern nur Versicherter wird. Zumal sich sowohl die gesetzliche Anknüpfung als auch die Beschränkung der Rechtswahl an subjektiven Kriterien des Versicherungsnehmers (gewöhnlicher Aufenthalt; Großrisiko) nicht aber des Versicherten ausrichtet, entsteht ein Schutzvakuum, das geschlossen werden muss. Wendete man die vorgestellten Entwürfe wörtlich an, so unterläge etwa eine zwischen einem deutschen Versicherer und einer deutschen Bank als Versicherungsnehmerin abgeschlossene Gruppenversicherung, die Risiken der Kreditkartenkunden der Bank als versicherten Personen deckt, deutschem Recht. Das würde auch für ausländische Bankkunden gelten, die der Gruppe als Mitglieder beitreten. Funktional betrachtet, tritt hier eine Schieflage ein, weil in diesen Fällen der Gruppenversicherung der Versicherte derjenige ist, der „Versicherung nimmt". Der Beitritt zur Gruppe, etwa durch Lösen einer Kreditkarte, ist funktional ${ }^{118}$ vom Abschluss eines eigenständigen Versicherungs-

116 Vgl. den geltenden Art. 13 Abs. 1 EGVVG.

117 ABl. 1992 Nr. L 228 / 1.

118 Im Gegensatz zur rechtlichen Konstruktion dieser Gruppenversicherungen, nach der die Bank bzw. das Kreditkartenunternehmen Versicherungsnehmer(in) ist; vgl. OLG Frankfurt/Main 9. 10.2002 VersR 2003, 361; auch öOGH 11. 5.1994 VersR $1995,443$. 
vertrags schwer abgrenzbar. Das Phänomen ist aus dem nationalen Recht bekannt. So hat die deutsche Bundesanstalt für Finanzdienstleistungsaufsicht bzw. ihr Vorläufer, das damalige Bundesaufsichtsamt für das Versicherungswesen, am 31.7.1990 das Rundschreiben R3/90119 herausgegeben, das gewisse Mindestanforderungen an Gruppenversicherungsverträge stellt, die das versicherte Gruppenmitglied rechtlich zumindest in die Nähe der Position eines Versicherungsnehmers rückt. Kollisionsrechtlich sollte hier nichts anderes gelten. Die EuGVVO bestätigt übrigens diesen Ansatz: Sie gewährt in Art. 9 Abs. 1 lit. b nicht nur dem Versicherungsnehmer, sondern auch dem Versicherten einen Heimatgerichtsstand. Dies trifft insbesondere auch den Fall eines im Rahmen einer Gruppenversicherung versicherten Gruppenmitglieds.

\subsubsection{Schutz des Gruppenmitglieds}

Art. 6 Abs. 3 lit a S 1 unterwirft bei objektiver Anknüpfung den Gruppenversicherungsvertrag dem Recht des Staates, in dem die Gruppenmitglieder ihren gewöhnlichen Aufenthalt haben. ${ }^{120}$ Dies erfordert konsequenterweise eine Vertragsspaltung, wenn sich der gewöhnliche Aufenthalt der Gruppenmitglieder in verschiedenen Staaten befindet (Art. 6 Abs. 3 lit. a S 2). Außerdem müssen die Voraussetzungen einer freien Rechtswahl entsprechend angepasst werden. Unbedenklich erscheint sie bei den sogenannten besonderen Risiken, die also nicht von subjektiven Voraussetzungen des Versicherungsnehmers bzw. Gruppenmitglieds abhängen. Bei der Definition des Großrisikos, die auch subjektive Kriterien enthält, aber auch bei der Definition des aktiv grenzüberschreitenden Nachfragers sind die Kriterien indessen so anzupassen, dass das einzelne Gruppenmitglied in den Genuss desselben Schutzes gelangt wie der Versicherungsnehmer nach Art. 6 Abs. 1. Diesem Zweck dient der vorgeschlagene Art. 6 Abs. 3 lit. b.

\subsubsection{Zusätzliche Rechtswahlmöglichkeit}

Die Rechtswahlmöglichkeit wird dann noch um einen speziellen Fall einer beschränkten Rechtswahl ergänzt. Schließt nämlich ein Arbeitgeber eine Gruppenversicherung für Risiken seiner Arbeitnehmer, so sollten die Treuepflichten des Arbeitgebers sicherstellen, dass die Interessen der Arbeitnehmer beim Abschluss des Versicherungsvertrags hinreichend berücksichtigt werden. Art. 6 Abs. 3 lit. c lässt daher eine Rechtswahl zugunsten des Rechts des Staates zu, in dem der Arbeitgeber seine Hauptniederlassung oder aber

119 VerBAV 1990, 339.

120 Vgl. insofern die geltende Regelung des Art. 13 Abs. 2 EGVVG, der eine Gruppenkrankenversicherung deutschem Recht unterwirft, wenn die versicherte Person ihren gewöhnlichen Aufenthalt in Deutschland hat. 
jene Betriebsstätte hat, an der oder von der aus der Arbeitnehmer seine Arbeit gewöhnlich verrichtet. Damit kann dem Interesse von Versicherer und die Versicherung nehmendem Arbeitgeber entsprochen werden, alle Versicherungsverhältnisse einem einzigen Recht $\mathrm{zu}$ unterstellen. Diese Rechtswahlmöglichkeit erscheint zugleich ungefährlich, weil alle Arbeitnehmer eine enge Beziehung zu diesem Ort haben.

\section{9 „Optionales europäisches Instrument“}

Der hier vorgestellte Vorschlag geht auf ein optionales europäisches Instrument nicht ein. Sein Art. 6 Abs. 2 nimmt nur auf die Möglichkeit einer Wahl nichtstaatlichen Rechts gemäß Art. 3 Abs. 2 des Kommissionsvorschlags Bezug, der aber - schon weil er im Massengeschäft die zwingenden Anknüpfungen nicht beseitigt - kein optionales Instrument schafft. Eine Regelung zum optionalen Instrument fehlt, weil ein solches in der Form einer EG-Verordnung erlassen und die Option in der Verordnung selbst vorgesehen werden sollte. ${ }^{121}$ Insofern bedarf es keiner Regelung im Rahmen der Rom-I-Verordnung. Der Bedarf nach einem solchen optionalen Instrument ist indessen zu unterstreichen. ${ }^{122}$

121 Ausführlich zur Ausgestaltung der Option Heiss / Downes, Non-Optional Elements in an Optional European Contract Law. Reflections from a Private International Law Perspective, ERPL 13 (2005), 693.

122 Siehe hierzu schon oben 2.1.2 und die Nachweise in FN 48 und 49. 